\title{
Risk factors for 30-day readmission in patients with ischemic stroke: a systematic review and meta-analysis
}

\author{
Zhiqiang Deng ${ }^{1}$, Xiaoyan $\mathrm{Wu}^{1}$, Linxue $\mathrm{Hu}^{1}$, Ming $\mathrm{Li}^{2}$, Muke Zhou ${ }^{2}$, Lihong Zhao ${ }^{3}$, Rong Yang ${ }^{2}$ \\ ${ }^{1}$ Department of Neurology, West China College of Nursing, Sichuan University/West China Hospital, Sichuan University, Chengdu, China; \\ ${ }^{2}$ Department of Neurology, West China Hospital of Sichuan University, Chengdu, China; ${ }^{3}$ Department of Radiology, West China Hospital, Sichuan \\ University, Chengdu, China \\ Contributions: (I) Conception and design: R Yang, L Zhao, M Zhou; (II) Administrative support: R Yang, M Zhou; (III) Provision of study materials or \\ patients: R Yang, L Zhao; (IV) Collection and assembly of data: Z Deng, X Wu, M Li; (V) Data analysis and interpretation: Z Deng, X Wu, L Hu; (VI) \\ Manuscript writing: All authors; (VII) Final approval of manuscript: All authors. \\ Correspondence to: Lihong Zhao. Department of Radiology, West China Hospital, Sichuan University, Chengdu, China. \\ Email: zhaolihong184@163.com; Rong Yang. Department of Neurology, West China Hospital, Sichuan University, Chengdu, China. \\ Email: hxyangrong2014@163.com.
}

Backgroundt The aim of this study was to identify risk factors for 30-day readmission in ischemic stroke
survivors, with an attempt to improve post-discharge care and lower the 30-day readmission rate.
Methods: Seven databases were searched from inception to April 30, 2021. Retrospective or prospective
observational studies and interventional studies focusing on 30-day readmission risk factors in patients with
ischemic stroke were included. Two authors independently screened the literature and evaluated the quality
of the studies using the Newcastle-Ottawa scale (NOS). The pooled effect size was estimated using the odds
ratio (OR), and the corresponding $95 \%$ confidence interval (CI) was calculated. The Cochrane Q $\left(\chi^{2}\right)$ and $\mathrm{I}^{2}$
tests were used to assess heterogeneity among studies, and each risk factor was tested for its robustness using
fixed- or random-effects models.

Results: A total of 17 retrospective observational studies from the United States $(\mathrm{n}=10)$, China $(\mathrm{n}=2)$, Republic of Korea ( $n=2)$, Norway ( $n=2)$, and Australia $(n=1)$, comprising a total of 1,829,964 patients, were included. The 30-day readmission rates of ischemic stroke survivors ranged from $1.41 \%$ to $27.64 \%$, with a mean value of $10.66 \% \pm 6.87 \%$. We finally identified 6 risk factors: history of stroke (OR, 1.33; $95 \%$ CI: 1.08 1.64; $\mathrm{P}=0.007)$, diabetes mellitus (OR, 1.15; 95\% CI: 1.13-1.17; $\mathrm{P}<0.001)$, hypertension (OR, 1.10; $95 \%$ CI: $1.07-1.13 ; \mathrm{P}<0.001)$, atrial fibrillation (OR, 1.26; 95\% CI: 1.23-1.29; $\mathrm{P}<0.001)$, heart failure (OR, 1.59; $95 \%$ CI: $1.56-1.63 ; \mathrm{P}<0.001)$, and age, among which age was determined by descriptive analysis. Four risk factors were ruled out: hyperlipidemia (OR, 1.01; 95\% CI: 0.87-1.17; P=0.91), coronary artery disease (OR, 0.83; 95\% CI: 0.73-0.96; P=0.009), smoking (OR, 0.97; 95\% CI: 0.83-1.14; P=0.71), and gender (female, OR, 0.97; 95\% CI: 0.96-0.98; $\mathrm{P}<0.001)$.

Discussion: The 30-day readmission rates of ischemic stroke survivors ranged from $1.41 \%$ to $27.64 \%$ and remained challenging. We found that stroke history, diabetes mellitus, hypertension, atrial fibrillation, heart failure, and advanced age were risk factors for 30-day readmission, whereas hyperlipidemia, coronary artery disease, smoking, and gender were not. All the studies included in this analysis were case-control studies, and thus causality cannot be inferred. Furthermore, recall bias may be present.

Keywords: Ischemic stroke; readmission; risk factors; meta-analysis

Submitted Sep 10, 2021. Accepted for publication Oct 22, 2021.

doi: 10.21037/apm-21-2884

View this article at: https://dx.doi.org/10.21037/apm-21-2884

\footnotetext{
^ ORCID: 0000-0001-9527-364X.
} 


\section{Introduction}

According to a report on the global burden of stroke between 1990 and 2016 (1), stroke is the second leading cause of death worldwide, second only to ischemic heart disease, accounting for nearly 50 million deaths annually. Stroke is a major public health problem throughout the world due to its high prevalence, mortality, disability rate, and incidence of complications. Stroke has a long disease course, and the recovery period ranges from 2 weeks to 6 months after the onset of the disease, with the sequelae period even continuing beyond 6 months. These facts make out-of-hospital management of stroke particularly important. However, the 30-day readmission rates in stroke survivors remain high due to a variety of factors, including disease recurrence, infection, limb dysfunction, and falls. In 2018, a Chinese study involving 50,912 stroke survivors from 375 hospitals in 29 provinces in China showed that $28.8 \%$ of patients were readmitted within 30 days of discharge (2). In a 6-year population-based cohort study of approximately 2 million adult stroke survivors in the United States, $13.7 \%$ of patients with hemorrhagic stroke, $12.4 \%$ of patients with acute ischemic stroke, and $11.5 \%$ of patients with subarachnoid hemorrhage were readmitted within 30 days (3). The high readmission rate reduces the quality of life of patients, causes considerable harm to patients and their families, and increases the medical and economic burden. It is also important to investigate the 15- or 60-day readmission rates of patients with ischemic stroke, but the study of Bjerkreim et al. (4) showed that 30-day readmission significantly increased the risk of 1-year mortality in patients surviving 30 days after discharge. Furthermore, the frequency of 30-day readmissions after stroke has become an indicator for the quality of care, quality of immediate post-discharge care, and the presence of a chronically ill and vulnerable population (5). Frequent readmissions can also affect hospital development and patient reimbursement (6). Vahidy et al. reported that $12.9 \%$ of 30 -day readmissions were preventable (7). Therefore, it is important to assess the risk factors for 30-day readmissions in ischemic stroke survivors and intervene accordingly.

Although many multicenter, retrospective, observational studies with large sample sizes have investigated the risk factors for 30-day readmission in patients with ischemic stroke in recent years, few prospective cohort studies, longitudinal studies, and randomized controlled trials (RCTs) have been published in this field. With diverse foci, these studies provide no definite conclusions and have not been properly summarized. No previous relevant metaanalyses (8-10) have specifically explored the stroke types and the timing of readmissions due to the limited number of the included articles and databases. Only a small number of risk factors have been identified, and many other risk factors warrant further investigation. Furthermore, these previous meta-analyses did not include several high-quality articles published in the past 5 years, and their conclusions need to be updated. The aim of this meta-analysis was therefore to identify the risk factors for 30-day readmission in ischemic stroke survivors, with an attempt to inform the out-of-hospital management of stroke and lower the 30-day readmission rate, thus benefiting more patients.

Whilst some researchers reported similar studies (9) in 2016, the highlight of our study is that we searched 3 Chinese databases, namely Wanfang data, China National Knowledge Infrastructure (CNKI), and Chinese Science and Technology Journal Database (VIP), and included excellent research in Chinese, rather than just research published in English, which previous similar institutes have not. We believe that research by Chinese scholars is essential to advance clinical work and scientific research. We have added 12 of the latest studies since 2016, and many of the studies are multicenter studies with large sample sizes, making the results more reliable. In addition, only 4 studies were included in previous studies to compare the differences between the experimental group and the control group, while 17 studies were included in our study, which is conducive to improving the persuasiveness of the results. Our study considered nearly 30 risk factors for 30-day readmission after ischemic stroke, and finally identified 6 risk factors after 4 risk factors were ruled out, with 5 possible risk factors requiring further investigations. We present the following article in accordance with the PRISMA reporting checklist (available at https://dx.doi. org/10.21037/apm-21-2884).

\section{Methods}

\section{Literature search}

Four English-language databases, including Web of Science (WOS), the National Library of Medicine (MEDLINE), Excerpta Medica Database (EMBASE), and the Cochrane Library, along with the 3 top Chinese-language databases, including Wanfang data, China National Knowledge Infrastructure (CNKI), and Chinese Science and Technology Journal Database (VIP), were searched from 
inception to April 30, 2021. Gray literature as well as the references of the relevant articles were also retrieved when possible. The search was conducted by using a combination of relevant subject headings and keywords including "stroke/ acute ischemic stroke/cerebral infarction/transient ischemic attack (TIA)/cerebral vascular accident" and "re*hospital*/ re*admission*”.

\section{Inclusion and exclusion criteria}

The inclusion criteria were as follows: (I) the article was a retrospective or prospective observational study or interventional study; (II) the subjects were patients with radiologically (cranial computed tomography or magnetic resonance imaging) confirmed ischemic stroke or identified according to the International Classification of Diseases, regardless of stroke type, lesion location, disease course, or comorbidity; (III) participants were aged $\geq 18$ years; and (IV) the study focused on risk factors for 30-day readmission in patients with ischemic stroke. The exclusion criteria were as follows: (I) studies that were not available in full text; (II) literature for which complete data were not available; (III) among repeated articles, the article had the least comprehensive data set; and (IV) review articles, case reports, and qualitative studies. Some of the articles on 30 -day unplanned readmissions or that focused on 28- or 31-day readmissions were also included, as we believed they were equally valuable for our analysis. We also included some articles that examined patients with different stroke types and articles in which patients with ischemic stroke accounted for more than $70 \%$ of all the participants. However, similar articles not involving ischemic stroke survivors or with a low proportion of ischemic stroke survivors were excluded. A few articles that did not appear suitable for our analysis were still included if we could find or calculate the data we needed from these articles, though information from these articles was typically quite limited.

\section{Data extraction and quality assessment}

Two researchers (DZQ and WXY) independently screened the literature based on the inclusion and exclusion criteria. Using a predesigned table, these 2 researchers independently extracted data including: (I) first author, publication year, country, study type, and sample size; (II) basic characteristics of the study population including age, stroke type, readmission time, readmission type, and 30-day readmission rate; and (III) outcome indicators including risk factors for 30-day readmissions. These 2 investigators also independently assessed study quality using methods developed by the US Agency for Healthcare Research and Quality (AHRQ), while the Newcastle-Ottawa Scale was applied for observational studies. Study quality scores were defined as poor [0-3], fair [4-6], or good [7-9]. Discrepancies encountered during literature screening, data extraction, and quality assessment were discussed and resolved in consultation with a third author (YR), if necessary. Where data were not available or were unclear from the reports, we contacted the corresponding authors for further information.

\section{Data synthesis and statistical analysis}

Review Manager version 5.3 (Cochrane Library) was used for outcome analysis. As all the included articles in the final analysis were retrospective observational studies, the pooled effect size was estimated using odds ratio (OR), and the $95 \%$ confidence interval (CI) was also calculated. A P value of $<0.05$ was considered statistically significant. Heterogeneity was assessed by the $\chi^{2}$ test and $\mathrm{I}^{2}$ value. If the heterogeneity of the study was acceptable $(\mathrm{P}>0.10$ and $\mathrm{I}^{2}<50 \%$ ), a fixed-effects model was applied, while the robustness of the test was validated using a random-effects model. If heterogeneity was present among the studies, the sources of heterogeneity were further analyzed. First, any possible errors that occurred during data extraction, recording, and input were checked for. Second, sensitivity analysis was performed to exclude studies that might have caused heterogeneity, which was followed by a reperforming of the meta-analysis to eliminate heterogeneity. If the heterogeneity was large $\left(\mathrm{P} \leq 0.10\right.$ and $\mathrm{I}^{2}>50 \%$ for outcome indicators) and the source of heterogeneity could not be determined, the meta-analysis could not be performed and only descriptive analysis was conducted. For missing or erroneous data in the original articles, we added them ourselves if they could be interpreted according to the main text. Otherwise, the corresponding authors of these articles were contacted for clarification via email. The search flowchart, basic information table, and quality evaluation table were created using Microsoft Word (Microsoft Corporation, Redmond, WA, USA), and the forest plots and inverted funnel plots were created using RevMan version 5.3. Publication bias was assessed using inverted funnel plots. 


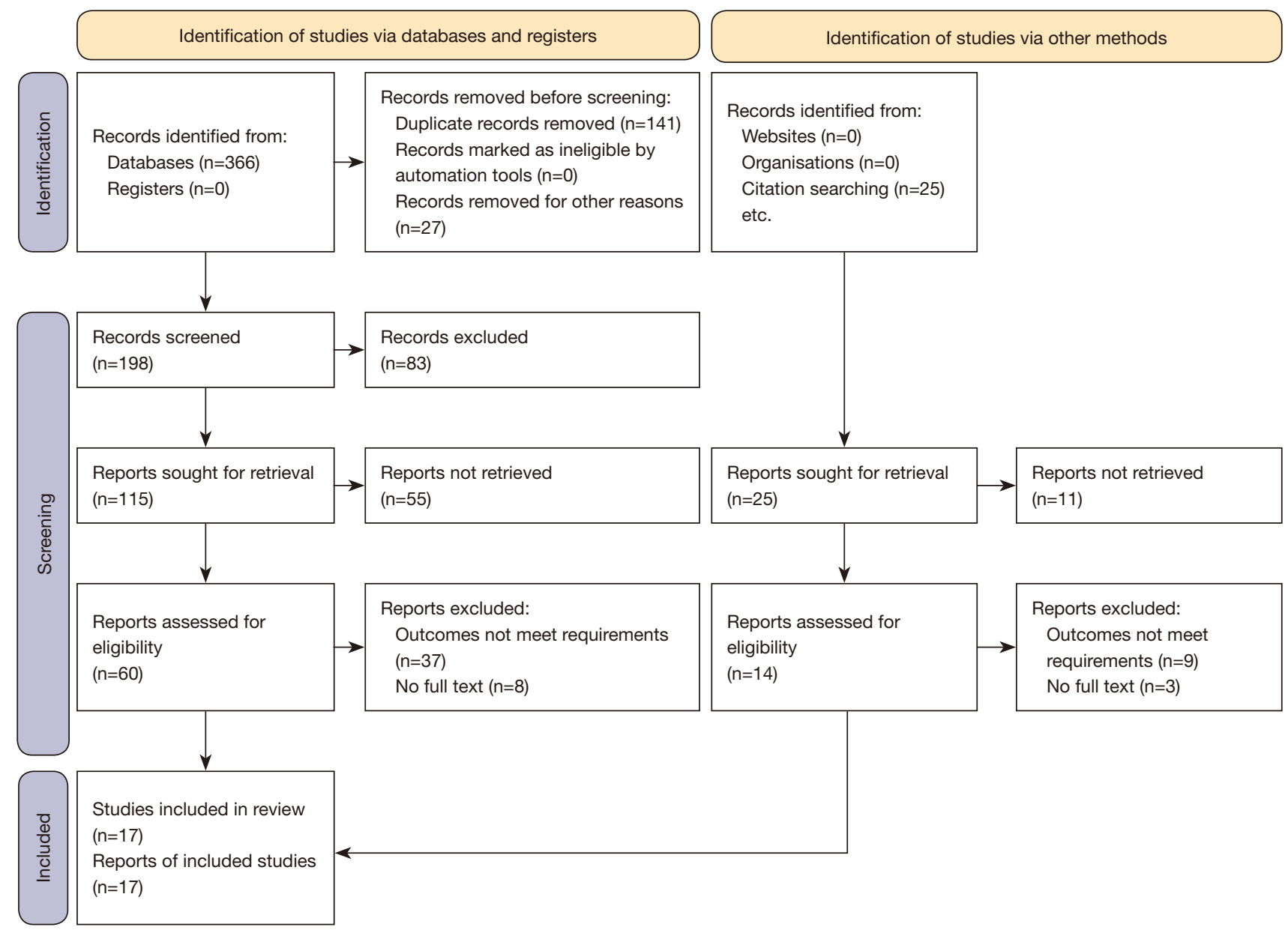

Figure 1 Flowchart of the review process.

\section{Results}

\section{Results of literature search}

A total of 366 articles were retrieved from the 7 databases, and 25 documents were found through other resources (Figure 1). Seventeen retrospective observational studies from the United States $(n=10)$, China $(n=2)$, Republic of Korea $(\mathrm{n}=2)$, Norway $(\mathrm{n}=2)$, and Australia $(\mathrm{n}=1)$, comprising a total of $1,829,964$ patients, were entered into the final meta-analysis. In contrast, the number of prospective observational and interventional studies (e.g., RCTs) was small. In addition, these articles covered a wide range of topics that did not focus on a specific number of risk factors. The number of articles addressing the same risk factor was $\leq 3$, and many factors were only described in just a single article. Thus, they were not deemed suitable for metaanalysis or descriptive systematic evaluation. The 30-day readmission rates of ischemic stroke survivors ranged from $1.41 \%$ to $27.64 \%$, with a mean value of $10.66 \%$ (SD $6.87 \%$ ). The results varied among countries and regions, with the highest reported 30-day readmission rate from China and the lowest from the United States (Table 1).

\section{Quality evaluation}

The included articles were of moderate quality $(\mathrm{n}=13)$ or high quality $(\mathrm{n}=4)$ (Table 2).

\section{Statistical analysis}

We screened about 30 of the most frequently mentioned risk factors in these 17 articles and finally identified 6 risk factors (Figures 2-6): history of stroke (OR, 1.33; 95\% CI: 1.08-1.64; $\mathrm{P}=0.007)$, diabetes mellitus (OR, 1.15; $95 \%$ 
Table 1 Characteristics of the 17 selected studies

\begin{tabular}{|c|c|c|c|c|c|c|c|}
\hline \multirow{2}{*}{ Studies } & \multirow{2}{*}{ Country } & \multirow{2}{*}{ Stroke type } & \multirow{2}{*}{$\begin{array}{l}\text { Readmission } \\
\text { time }\end{array}$} & \multirow{2}{*}{$\begin{array}{l}\text { Readmission } \\
\text { type }\end{array}$} & \multicolumn{2}{|c|}{ Readmission $(\mathrm{N})$} & \multirow{2}{*}{ Effective factors, OR (95\% Cl) } \\
\hline & & & & & Yes & No & \\
\hline uet al. & & IS & $30 \mathrm{~d}$ & All causes & $\begin{array}{c}504 \\
22.96 \%\end{array}$ & 1,691 & $\begin{array}{l}\text { Age, } 1.04 \text { (1.03-1.05); NIHSS on admission, } 1.03 \text { (1.00-1.05); prior stroke, } 1.36 \text { (1.07-1.7 } \\
\text { ndwelling urinary catheter, } 1.53 \text { (1.13-2.07); on non-neurology floor, } 1.45 \text { (1.10-1.91) }\end{array}$ \\
\hline
\end{tabular}

2021

$(85 \%) ; ~ H S$
$(15 \%)$

ental. Republic IS (75.6\%);

2019 of Korea HS $(24.4 \%$

Wen et al. China IS

2019

Berkreim - Norway IS (89.60\%)

TIA $(10.40 \%)$

$30 \mathrm{~d}$

et al. 2018

Boehme
et al. 2018

Crispo
2018

USA

is

$30 \mathrm{~d}$

USA IS (22.96\%) $(77.04 \%)$ indwelling urinary catheter, 1.53 (1.13-2.07); on non-neurology floor, $1.45(1.10-1.91)$

$30 \mathrm{~d}$

$$
\begin{array}{rr}
27,161 & 203,708 \\
(11.76 \%) & (88.24 \%)
\end{array}
$$
$1.26(1.08-1.47)$

2,513

Age: occupation (employees of enterprises and institutions, workers, farmers, unemployed, retirees, others); payment application of surgery; discharge mode (medical discharge or transfer, nonmedical discharge)

Unplanned $200 \quad 1,674$ Age (years), mean \pm SD, 1.02 (1.01-1.03); NIHSS score at discharge, median (IQR); BI score at discharge, median (IQR) (10.67\%) (89.33\%) stroke subtype (large artery atherosclerosis), 1.74 (1.20-2.51); stroke subtype (small vessel occlusion); stroke subtype (undetermined etiology); peripheral arterial disease, 1.58 (1.01-2.47); Angina pectoris; hypertension; risk factor burden; (1.11-3.11); seizures; any complication; discharge destination (home; nursing home; other departmen

All causes $\quad 48,125 \quad 323,462 \quad$ Urinary tract infection, $1.11(1.06-1.17)$

$(12.95 \%)(87.05 \%)$

All causes $\quad 6,205 \quad 433,477$ Age $(<40,40-49,50-59,60-69,70-79,80-89,90+), 1.12$ (1.00-1.26): primary payer: private insurance; medicare, 1.33
$4,124 \quad 40,605$ Age: $18-44,1 ; 45-65,0.93$ (0.81-1.07); 65-75, 1.03 (0.89-1.19); 75+, 1.03 (0.89-1.20); type of insurance: NHI, 1; medical aid, Gender,

1.16 (1.03-1.30); length of stay of the index admission: less than 7 days, $1 ; 7-14$ days, 1.1 (1.01-1.19; More than 15 days, 1.34 (1.00-1.95): hospital region: capital area, 1; metropolitan area, 1.21 (1.07-1.37); nonmetropolitan area method of medical expenses (basic medical insurance for urban workers, basic medical insurance for urban residents, new
rural cooperative medical care, public expense, self-financed, other social insurance, others): grade of hospital (grade 2,3 High blood pressure: abnormal lipid metabolism; heart-related diseases; length of hospital stay; use of clinical pathways; complications during the stroke hospitalization (urinary tract infection; urinary retention; pneumonia; enteral feeding, 1.86

(1.41\%) (98.59\%) (1.26-1.40): Medicaid 1.41 (1.32-1.51): self-pay, 1.04 (0.93-1.16): no charge, 1.01 (0.72-1.41): median household income

$\$ 66,000+: \$ 51,000-\$ 65.999,0.97(0.92-1.02): \$ 40,000-\$ 50,999,1.01(0.96-1.06): \$ 1-\$ 39,999,1.08$ (1.03-1.14): length of stay: 0-7 days; > 7 days, 1.38 (1.33-1.43); discharge disposition: routine; transfer: short-term hospital, 1.91 (1.70-2.14); transfer: other type of facility, 1.52 (1.45-1.59); home health care, 1.26 (1.20-1.32); against medical advice, $2.41(2.08-2.79)$; discharged alive, destination unknown, 0.20 (0.11-0.38), comorbidities: 0-2; 3-4, 1.36 (1.30-1.43); 5-6, 1.78 (1.68-1.87); 7 t 2.20 (2.08-2.34); bed size of hospital: small; medium, 1.04 (0.98-1.11); large, 1.08 (1.02-1.15); control/ownership of hospital: government, non-federal (public); private, not-for-profit (voluntary), 1.04 (0.97-1.11); private, investor owned (proprietary), 1.20

$\begin{array}{lrl}\text { Khanevski } & \text { Norway } & \text { IS }(89.01 \%) \\ \text { et al. } 2018 & \text { TIA }(10.99 \%)\end{array}$

Bl score, median (IQR); Index etiology (TOAST): large-artery atherosclerosis (LAA), 4.36 (2.01-9.47); Index etiology (TOAS (1)
Allen et
2017
USA $\begin{array}{ccc}\text { All causes } & 57 & 359 \\ & (13.70 \%) & (86.30 \%)\end{array}$ Coronary artery disease; diabetes

Invalid factors

*Discharge destination (rehabilitation), 0.81 (0.66-0.99). *Discharge destination (Nursing home), 1.30 (1.02-1.65). Females; males; $\mathrm{BMl} \geq 24 \mathrm{~kg} / \mathrm{m}^{2}$; length of stay; hypertension; current smoking; current drinking, hyperlipidemia; atrial fibrillation; coronary artery disease family history of stroke; nasogastric tube feeding; reimbursement of medical insurance $20 \%$; thrombolytic therapy; thrombectomy; thrombolytic therapy + thrombectomy

\section{(nder; admiss}

Female; marital status (married, other); route of admission [emergency, outpatient, others

"mRS score at discharge, median (IQR), 0.99 (0.89-1.10); male sex; stroke subtype (cardioembolism); stroke subtype (other determined etiology); prior stroke; diabetes; myocardial infarction; atrial fibrillation; prior/current smoking; treatment (intravenous thrombolysis); treatment (thrombectomy); complications during the stroke hospitalization (incontinence; stroke in progression); length of stay, median (IQR); discharge destination Sepsis; pneumonia

Sex

Age (years) mean $\pm \mathrm{SD}$; sex (male); mRS score, median (IQR); NIHSS score, median (IQR); Index etiology (TOAST): small vessel disease; Index etiology (TOAST): undetermined; diabetes; angina pectoris; myocardial infarction; hypertension; atrial fibrillation; prior/ current smoking; treatment: IV thrombolysis; discharged to home; discharged to Home
nursing; discharged to Rehabilitation; discharged to Nursing home Not evaluated within 21 days; smoking history; dyslipidemia; atrial fibrillation; hypertension; initial admission NIHSS; Neurology consultation; gender; ethnicity; discharge disposition; employment status; insurance type; IV tPA 
Table 1 (continued)

\begin{tabular}{|c|c|c|c|c|c|c|c|c|}
\hline \multirow{2}{*}{ Studies } & \multirow{2}{*}{ Country } & \multirow{2}{*}{ Stroke type } & \multirow{2}{*}{$\begin{array}{l}\text { Readmission } \\
\text { time }\end{array}$} & \multirow{2}{*}{$\begin{array}{l}\text { Readmission } \\
\text { type }\end{array}$} & \multicolumn{2}{|c|}{ Readmission (N) } & \multirow{2}{*}{ Effective factors, OR (95\% Cl) } & \multirow{2}{*}{ Invalid factors } \\
\hline & & & & & Yes & No & & \\
\hline $\begin{array}{l}\text { Mittal et al. } \\
2017\end{array}$ & USA & Is & $30 \mathrm{~d}$ & unplanned & $\begin{array}{c}35 \\
(6.90 \%)\end{array}$ & $\begin{array}{c}472 \\
(93.10 \%)\end{array}$ & $\begin{array}{l}\text { Married at presentation, } 0.47 \text { (0.18-1.14); Educational level (high school graduation or higher), } 0.43 \text { (0.16-1.02); married at } \\
\text { presentation; living arrangement (assisted living), 2.25 (0.63-7.11); hypertension, } 4.72 \text { (0.79-92.3); dementia, 2.55 (0.76-8.52); } \\
\text { discharge disposition after index stroke (Nursing home), } 0.29 \text { (0.08-0.84) }\end{array}$ & $\begin{array}{l}\text { Age in years (mean } \pm \text { SD); sex; living arrangement (apartment/house; nursing home; } \\
\text { other); smoking (current smokers; past smokers; never smoked); previous IS; intracranial } \\
\text { hemorrhage; coronary artery disease; atrial fibrillation; hearlt failure; hyperlipidemia; } \\
\text { diabetes; intravenous thrombolysis; median length of stay (days); discharge disposition } \\
\text { after index stroke (home; rehabilitation) }\end{array}$ \\
\hline $\begin{array}{l}\text { Nouh et al. } \\
2017\end{array}$ & USA & $\begin{array}{l}\text { IS (67\%); HS } \\
(22 \%) ; \mathrm{TIA} \\
(11 \%)\end{array}$ & $30 \mathrm{~d}$ & All causes & $\begin{array}{c}134 \\
(8.63 \%)\end{array}$ & $\begin{array}{c}1,418 \\
(91.37 \%)\end{array}$ & $\begin{array}{l}\text { Age }>75,1.18 \text { ( } 0.77-1.81) \text {; residence in facility, } 1.41(0.75-2.68) \text {; prior stroke, } 1.39 \text { (0.91-2.12); diabetes mellitus, } 1.26 \\
\text { (0.85-1.87); chronic heart failure, } 1.63(0.99-2.67) \text {; atrial fibrillation, } 1.26(0.80-1.99) \text {; admit to non-neurology service, } 2.04 \\
\text { (1.28-3.27); on non-neurology floor, } 1.10 \text { (0.72-1.68) }\end{array}$ & $\begin{array}{l}\text { Male gender; living without spouse; obese (BMI >30); high cholesterol; hypertension; } \\
\text { depression; dementia; HS }\end{array}$ \\
\hline $\begin{array}{l}\text { Vahidy et al. } \\
2017\end{array}$ & USA & is & $30 \mathrm{~d}$ & All causes & $\begin{array}{c}38,625 \\
(12.10 \%)\end{array}$ & $\begin{array}{l}280,692 \\
(87.90 \%)\end{array}$ & 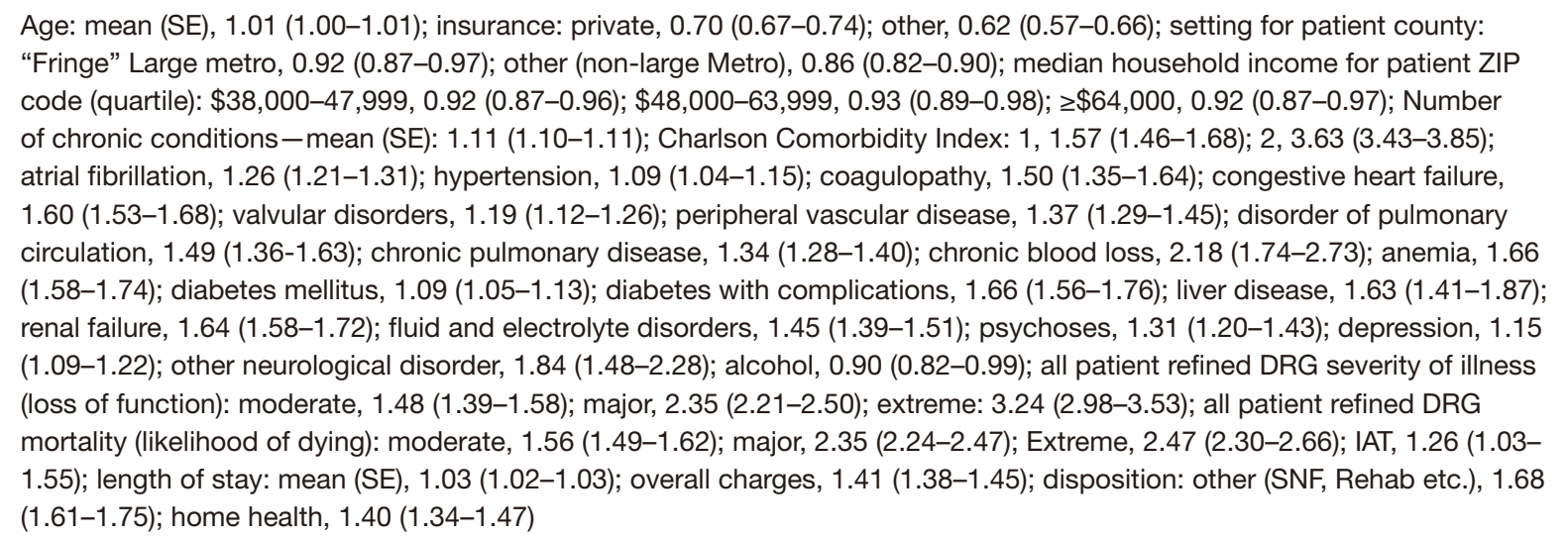 & $\begin{array}{l}\text { Female; insurance: Medicaid; ulcer; Alzheimer's disease; drug abuse; obesity; IV tPA; IV } \\
\text { tPA and/or IAT; admitted on a weekend }\end{array}$ \\
\hline $\begin{array}{l}\text { Han et al. } \\
2015\end{array}$ & $\begin{array}{l}\text { Republic } \\
\text { of Korea }\end{array}$ & $\begin{array}{c}\mathrm{ICH} \\
(19.71 \%) ; \mathrm{Cl} \\
(80.29 \%)\end{array}$ & $30 \mathrm{~d}$ & All causes & $\begin{array}{c}1,782 \\
(1.79 \%)\end{array}$ & $\begin{array}{c}97,682 \\
(98.21 \%)\end{array}$ & 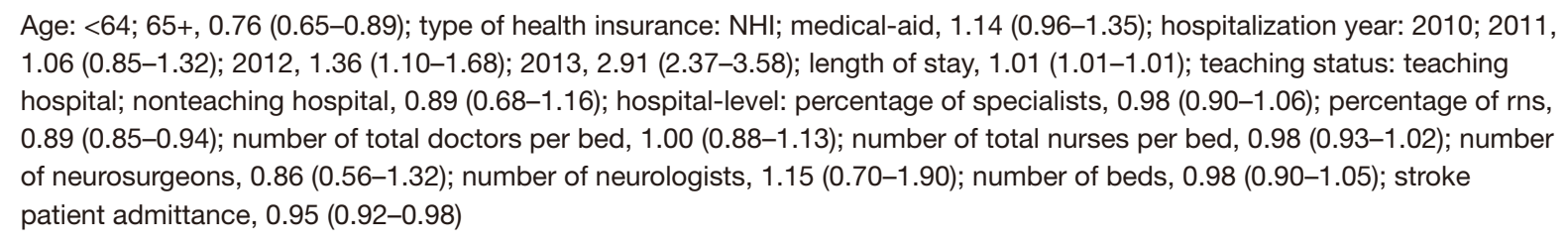 & Gender; Charlson Comorbidity Index $(0 ; 1 ; 2 ; 3+)$; hospital ownership (public; private) \\
\hline $\begin{array}{l}\text { Kilkenny } \\
\text { et al. } 2013\end{array}$ & Australia & $\begin{array}{l}\text { IS (91.41\%); } \\
\text { HS (8.59\%) }\end{array}$ & $28 d$ & All causes & $\begin{array}{c}215 \\
(6.46 \%)\end{array}$ & $\begin{array}{c}3,113 \\
(93.54 \%)\end{array}$ & $\begin{array}{l}\text { Dependent before admission (mRS, 2-5), } 1.87 \text { (1.25-2.81); ischemic heart disease, } 1.36 \text { (0.92-2.02); incontinent <72-h } \\
\text { admission, } 1.19 \text { ( } 0.77-1.83) \text {; health system: rural hospital; no CT scan or MRI (<24 h), } 1.78(1.00-3.14) \text {; health outcomes: } \\
\text { dependent at discharge (mrs, 3-5); any severe complication, } 2.81 \text { (1.55-5.12) }\end{array}$ & 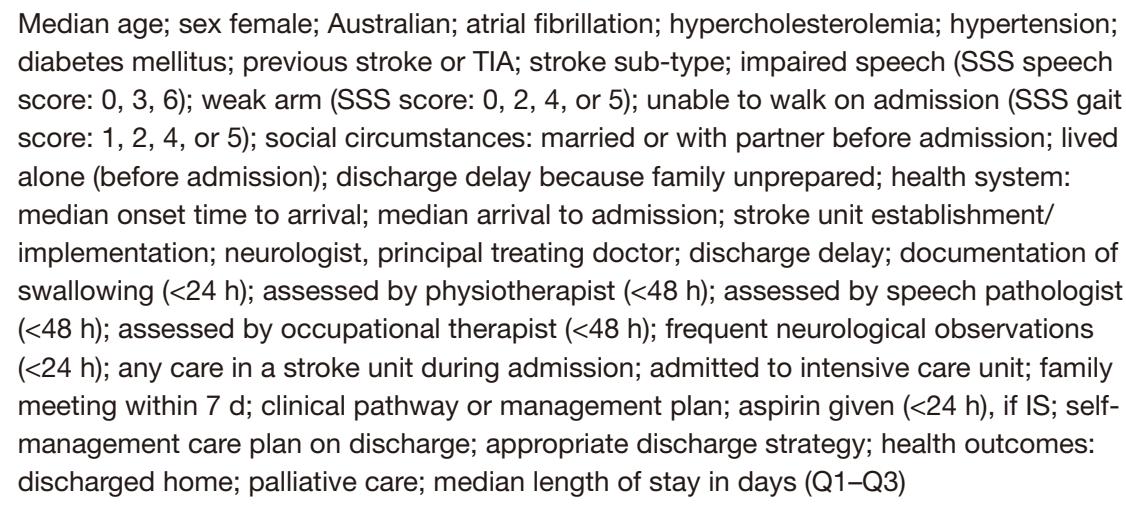 \\
\hline $\begin{array}{l}\text { Lichtman } \\
\text { et al. } 2013\end{array}$ & USA & Is & $30 \mathrm{~d}$ & All causes & $\begin{array}{c}44,379 \\
(14.41 \%)\end{array}$ & $\begin{array}{l}263,508 \\
(85.59 \%)\end{array}$ & 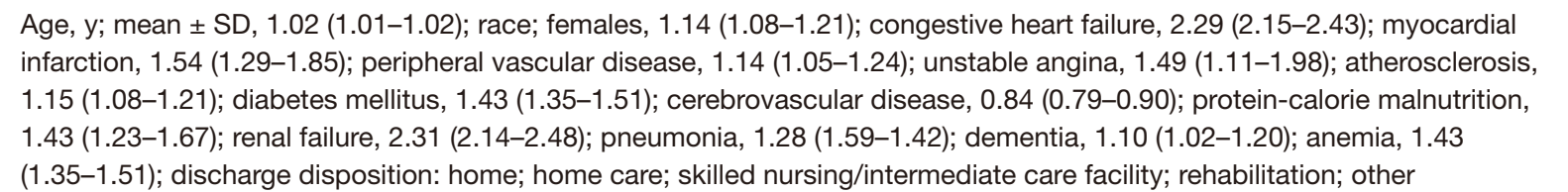 & - \\
\hline
\end{tabular}


Table 1 (continued)

\begin{tabular}{|c|c|c|c|c|c|c|c|}
\hline \multirow{2}{*}{ Studies } & \multirow{2}{*}{ Country } & \multirow{2}{*}{ Stroke type } & \multirow{2}{*}{$\begin{array}{l}\text { Readmission } \\
\text { time }\end{array}$} & \multirow{2}{*}{$\begin{array}{l}\text { Readmission } \\
\text { type }\end{array}$} & \multicolumn{2}{|c|}{ Readmission $(\mathrm{N})$} & \multirow{2}{*}{ Effective factors, OR (95\% Cl) } \\
\hline & & & & & Yes & No & \\
\hline $\begin{array}{l}\text { Suri et al. } \\
2013\end{array}$ & USA & is & $30 \mathrm{~d}$ & All causes & $\begin{array}{c}90 \\
(8.84 \%)\end{array}$ & $\begin{array}{c}928 \\
(91.16 \%)\end{array}$ & Age; diabetes mellitus; discharge disposition (home with health services; rehabilitation facility; short-term facility \\
\hline $\begin{array}{l}\text { Bhattacharya } \\
\text { et al } 2011\end{array}$ & USA & $\begin{array}{l}\text { IS (77.34\%); } \\
\text { TIA (22.66\%) }\end{array}$ & $30 \mathrm{~d}$ & All causes & $\begin{array}{c}22 \\
(11.46 \%)\end{array}$ & $\begin{array}{c}170 \\
(88.54 \%)\end{array}$ & Congestive heart failure; coronary artery disease; NIHSS $\geq 10$; discharge destination (home/acute rehabilitation) \\
\hline
\end{tabular}

Invalid factors

Sex; Race (White; African American; other); insurance; smoking status (current smoker; past smoker; never smoked); hypertension; hyperlipidemia; wake forest scale (mild stroke; moderate stroke; severe stroke; unknown); hours from symptom onset to arrival in emergency department $(<1 \mathrm{~h} ; 1-2 \mathrm{~h} ; 2-3 \mathrm{~h} ;>3 \mathrm{~h})$; previous history of stroke; thrombolytics administered; Neurology consulted

Mean age; male sex; race (African American; White; Hispanic; others); insurance (Medicare/ Medicaid; private insurers; uninsured); current smokers; cocaine; hypertension; atrial atherosclerosis; cardioembolic; small vessel disease; other unknown); treatments offered (iv TPA; Intervention; Aspirin; Statin)

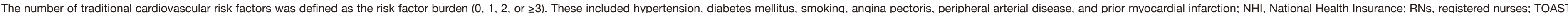

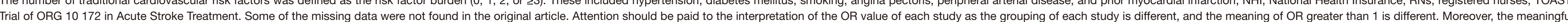

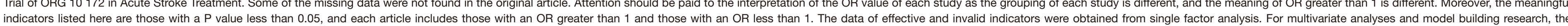

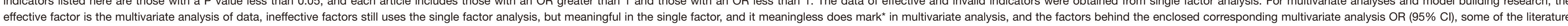

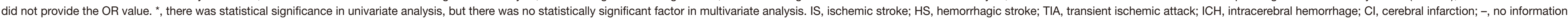
Bl, Barthel Index; IQR, interquartile range; mRS, modified Rankin Scale; NIHSS, National Institutes of Health Stroke Scale; SD, standard deviation. 


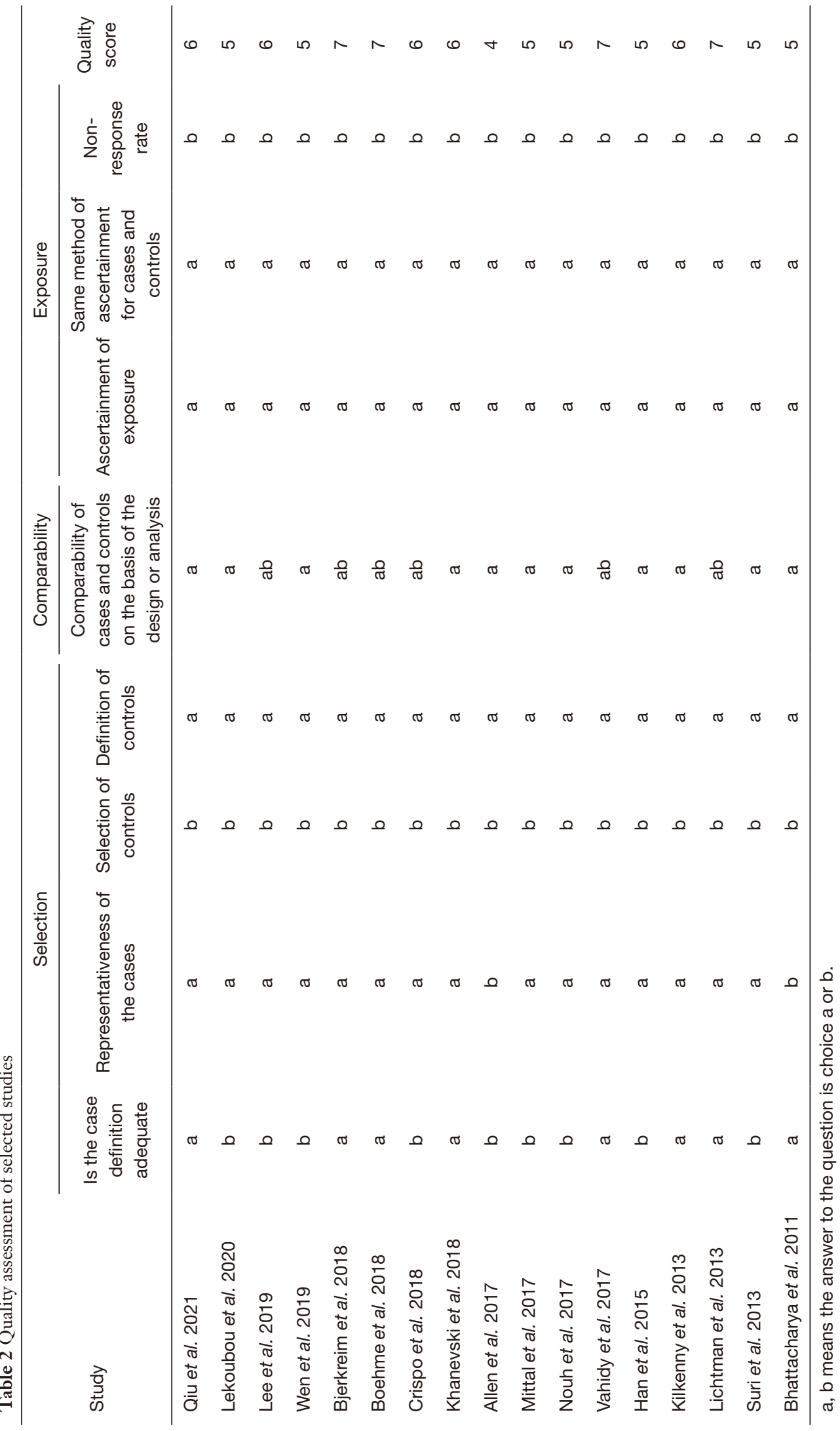




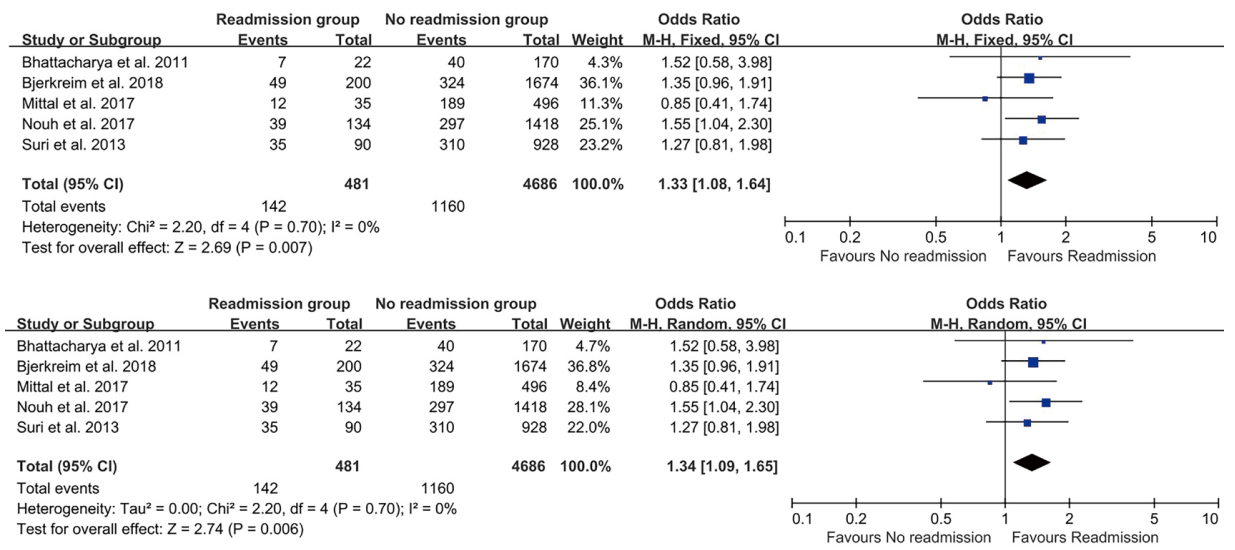

Figure 2 Influence of prior stroke on 30-day readmission in patients with ischemic stroke (Fixed effects model, Random effects model).

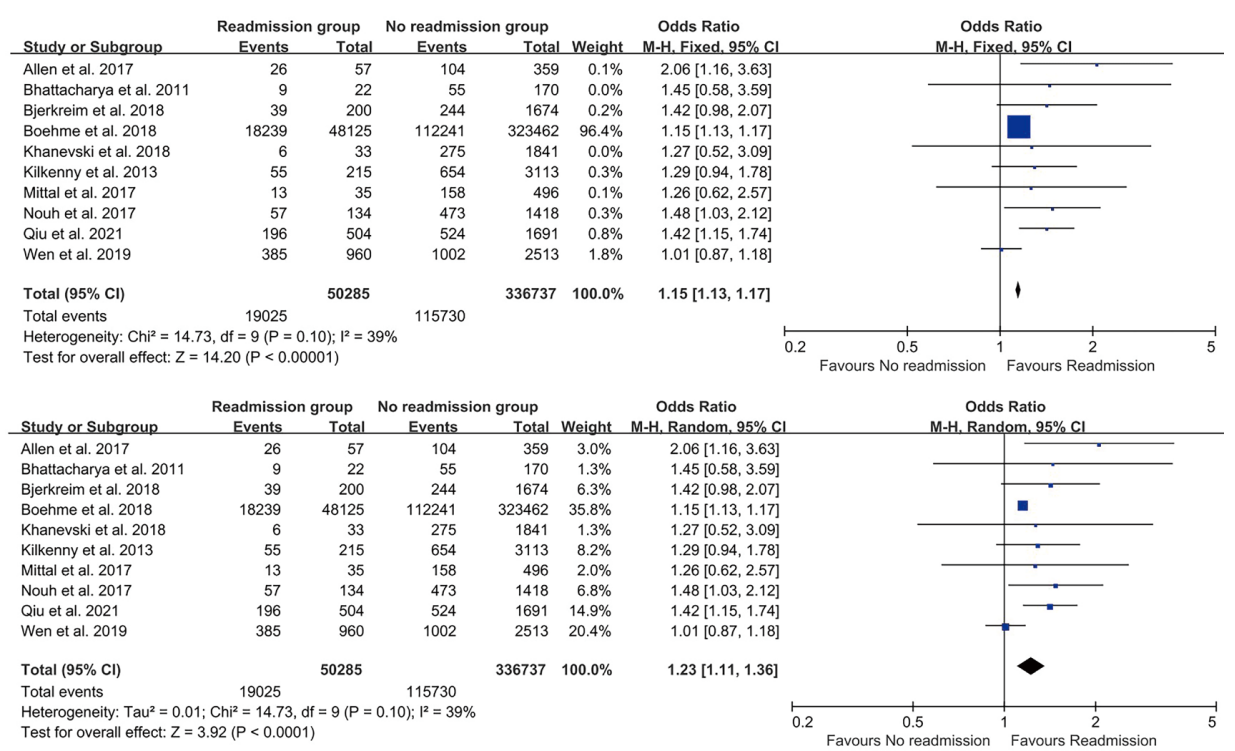

Figure 3 Influence of diabetes mellitus on 30-day readmission in patients with ischemic stroke (Fixed effects model, Random effects model).

CI: $1.13-1.17 ; \mathrm{P}<0.001)$, hypertension $(\mathrm{OR}, 1.10 ; 95 \%$ CI: $1.07-1.13 ; \mathrm{P}<0.001)$, atrial fibrillation $(\mathrm{OR}, 1.26 ; 95 \%$ CI: $1.23-1.29 ; \mathrm{P}<0.001)$, heart failure (OR, 1.59; $95 \%$ CI: $1.56-1.63 ; \mathrm{P}<0.001)$, and age, among which age was determined by descriptive analysis. Four risk factors were ruled out: hyperlipidemia (OR, 1.01; 95\% CI: 0.87-1.17; $\mathrm{P}=0.91)$, coronary artery disease $(\mathrm{OR}, 0.83 ; 95 \% \mathrm{CI}: 0.73-$ 0.96; $\mathrm{P}=0.009$ ), smoking (OR, 0.97; 95\% CI: 0.83-1.14; $\mathrm{P}=0.71$ ), and gender (female, OR, 0.97; 95\% CI: 0.96-0.98; $\mathrm{P}<0.001$; Figures $7-10$ ). Five possible risk factors requiring further investigation included duration of hospitalization, treatment modality (thrombolysis and thrombectomy), discharge disposition (home, rehabilitation facility, nursing home, home nursing, and others), health care payment model (Medicare, NHS, Medicaid, private insurance, and others), and etiology (atherosclerosis, cardiogenic cerebral embolism, small vessel disease, other definite causes, and other unknown causes). Intravenous thrombolysis (OR, 0.93; 95\% CI: 0.91-0.96; $\mathrm{P}<0.001)$ and post-discharge rehabilitation (OR, 0.78 ; $95 \%$ CI: $0.65-0.93 ; \mathrm{P}=0.007)$ were protective factors for 30-day readmissions in ischemic stroke survivors (Figures 11-14). Due to insufficient data reported in the studies, 17 factors could not be assessed, including epilepsy, peripheral arterial disease, deep vein thrombosis, dementia, infection, obesity, hospital region, ethnicity, renal failure, depression, admission to a non-neurological 


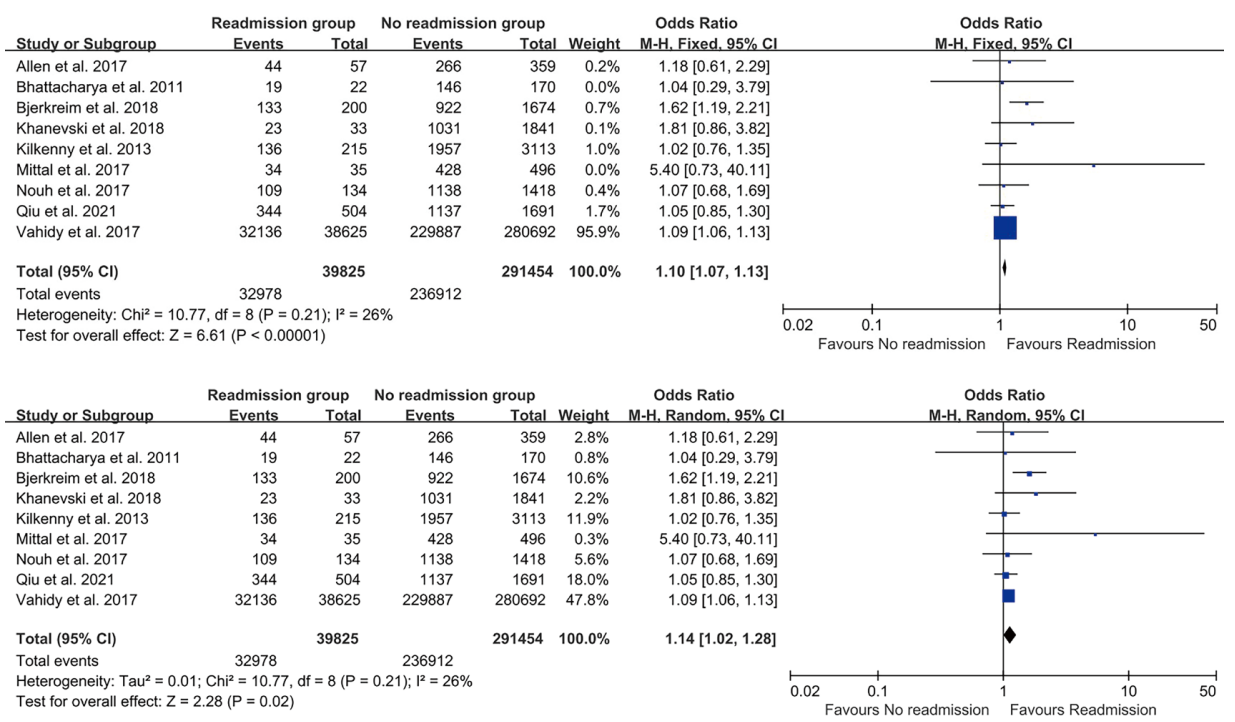

Figure 4 Influence of hypertension on 30-day readmission in patients with ischemic stroke (Fixed effects model, Random effects model).

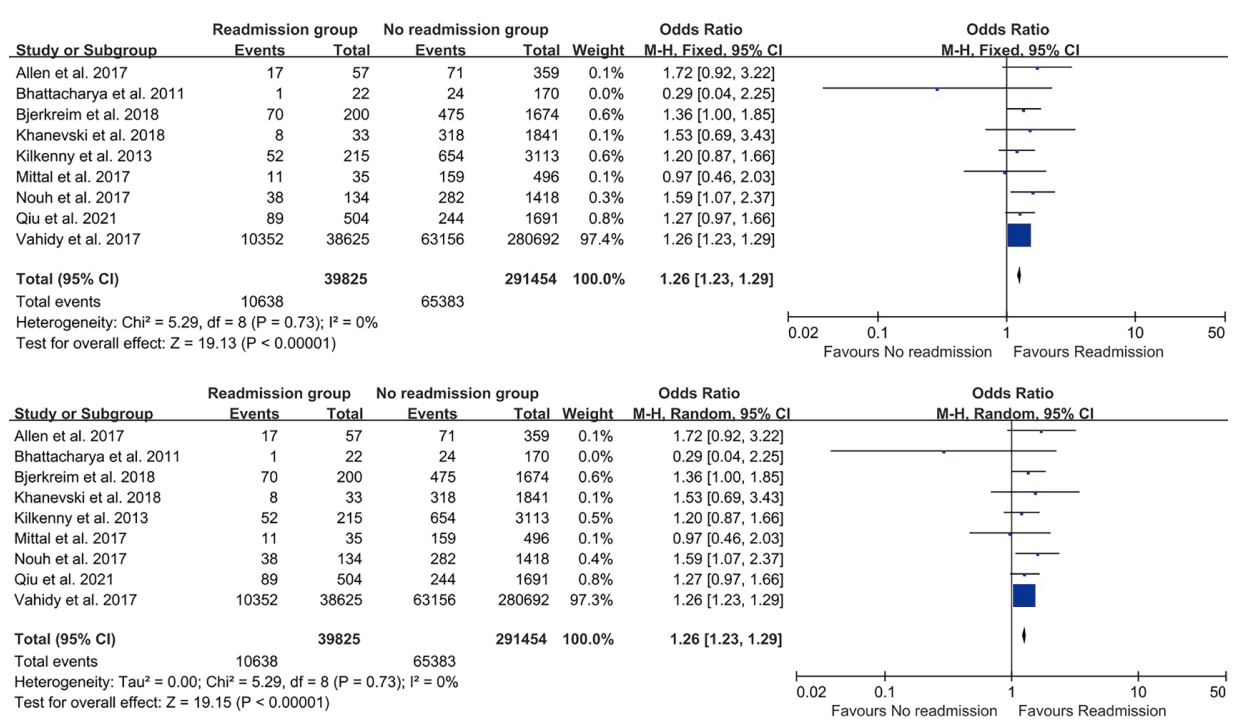

Figure 5 Influence of atrial fibrillation on 30-day readmission in patients with ischemic stroke (Fixed effects model, Random effects model).

department, admission to an emergency department, admission on weekends, alcohol consumption, marriage, admission to a teaching hospital, and hospital ownership. Sensitivity analysis was performed for all analysis results, and there was no significant heterogeneity. Heterogeneity might have arisen from differences in geography, sample size, and study population across studies.

\section{Publication bias}

Inverted funnel plots for assessing publication bias are shown in Figures 15-27. Except for diabetes mellitus (Figure 16), hyperlipidemia (Figure 17), and intravenous thrombolysis (Figure 24), which might have involved some publication bias, none of the other factors showed 


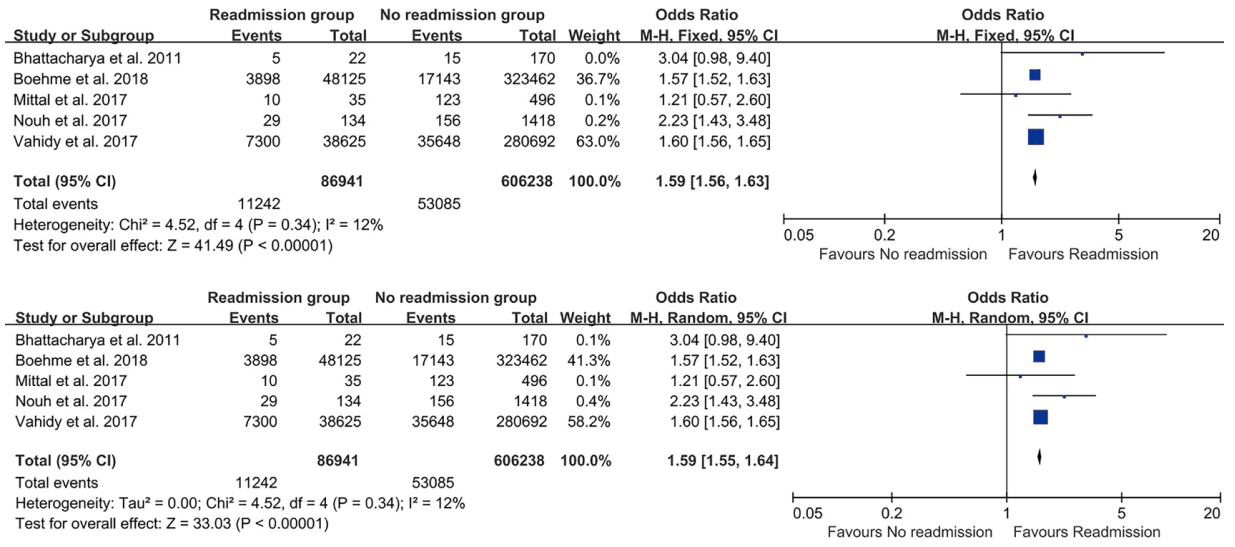

Figure 6 Influence of heart failure on 30-day readmission in patients with ischemic stroke (Fixed effects model, Random effects model).

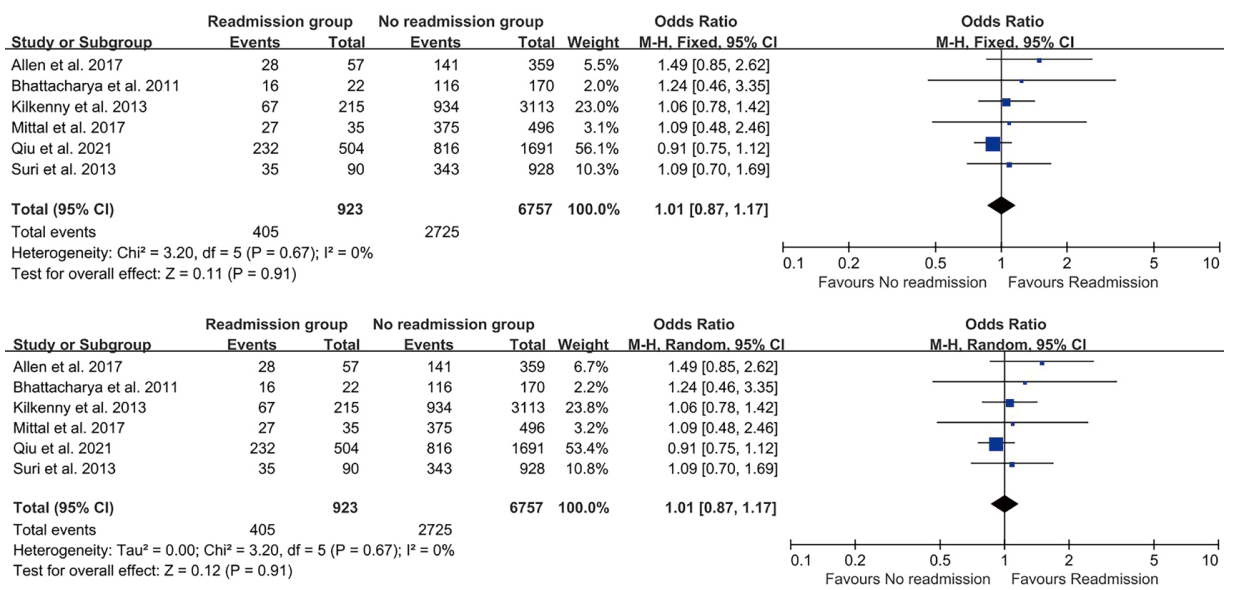

Figure 7 Influence of hyperlipidemia on 30-day readmission in patients with ischemic stroke (Fixed effects model, Random effects model).

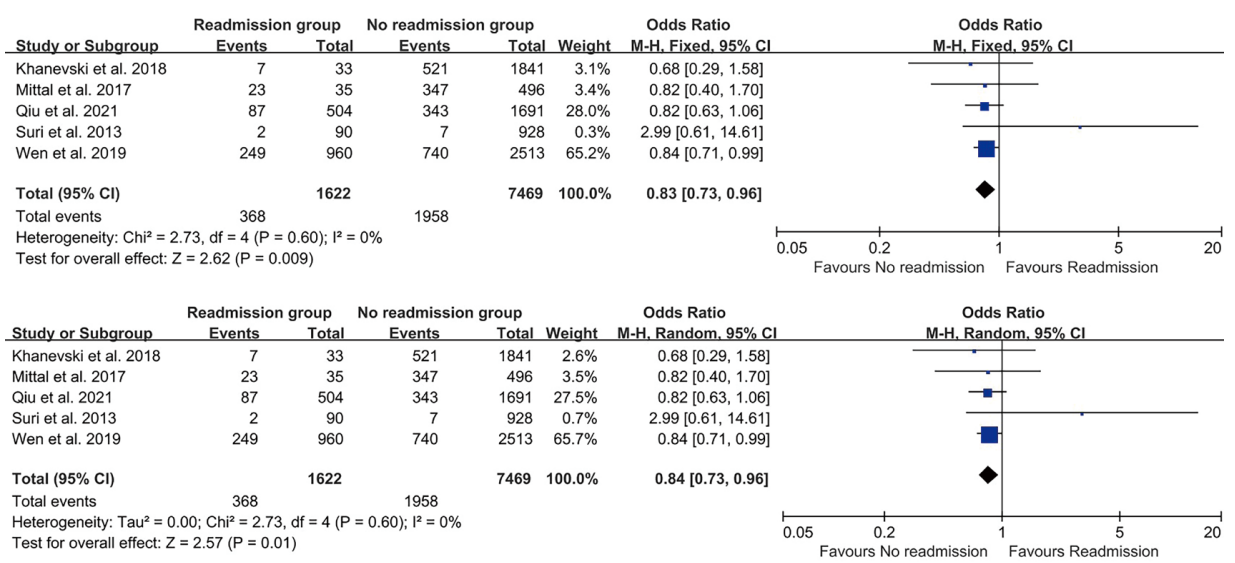

Figure 8 Influence of coronary heart disease on 30-day readmission in patients with ischemic stroke (Fixed effects model, Random effects model). 


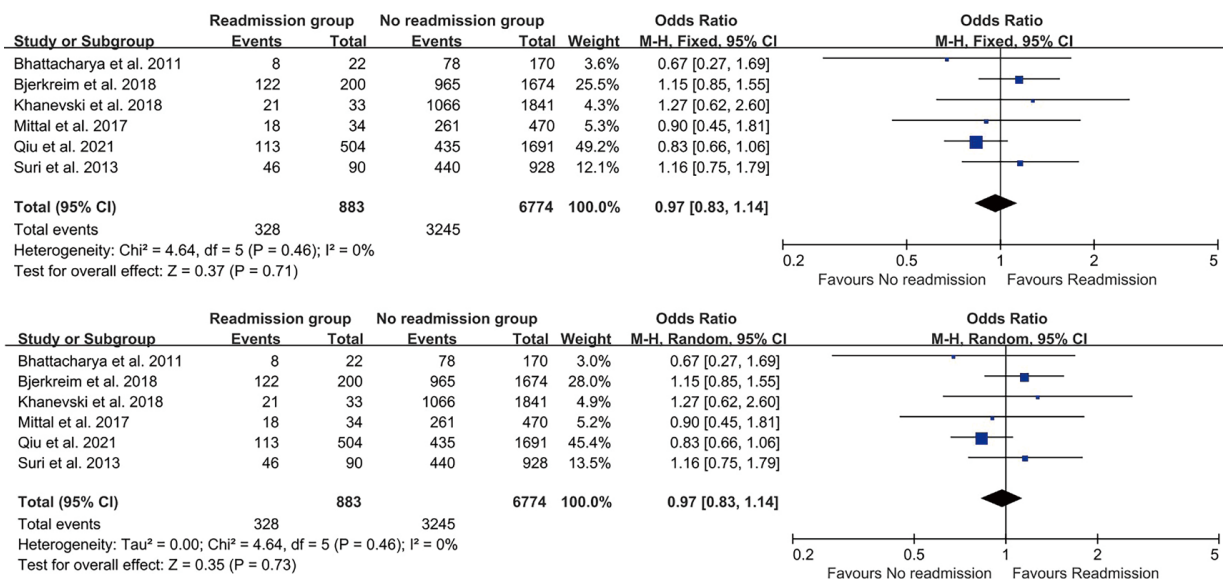

Figure 9 Influence of smoking on 30-day readmission in patients with ischemic stroke (Fixed effects model, Random effects model).

\begin{tabular}{|c|c|c|c|c|c|c|c|c|c|c|}
\hline \multirow{2}{*}{ Study or Subgroup } & \multicolumn{2}{|c|}{ Readmission group } & \multicolumn{2}{|c|}{ No readmission group } & \multirow{2}{*}{\multicolumn{3}{|c|}{$\begin{array}{c}\text { Odds Ratio } \\
\text { - }\end{array}$}} & \\
\hline & Events & \multirow{2}{*}{$\frac{\text { Total }}{22}$} & Events & Total & & & & \multicolumn{3}{|c|}{$\begin{array}{c}\text { Odds Ratio } \\
\text { M-H. Fixed. } 95 \% \mathrm{Cl} \\
\end{array}$} \\
\hline Bhattacharya et al. 2011 & 8 & & 79 & 170 & $0 \quad 0.0 \%$ & $0.66[0.26,1.65]$ & & 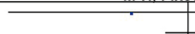 & & \\
\hline Bjerkreim et al. 2018 & 95 & 200 & 743 & 1674 & $0.2 \%$ & $1.13[0.85,1.52]$ & & \multirow{2}{*}{ I } & & \\
\hline Boehme et al. 2018 & 24592 & 48125 & 168200 & 323462 & $47.1 \%$ & $0.96[0.95,0.98]$ & & & & \\
\hline Crispo et al. 2018 & 3024 & 6205 & 222546 & 433477 & $7.1 \%$ & $0.90[0.86,0.95]$ & & - & & \\
\hline Han et al. 2015 & 806 & 1782 & 44072 & 97682 & $1.9 \%$ & $1.00[0.91,1.10]$ & & & - & \\
\hline Khanevski et al. 2018 & 16 & 33 & 822 & 1841 & $0.0 \%$ & $1.17[0.59,2.32]$ & & & \multirow[b]{2}{*}{ - } & \\
\hline Kilkenny et al. 2013 & 101 & 215 & 1532 & 3113 & $0.2 \%$ & $0.91[0.69,1.21]$ & & & & \\
\hline Lee et al. 2019 & 1836 & \multirow{2}{*}{$\begin{array}{r}4124 \\
35\end{array}$} & 17819 & 40605 & $4.0 \%$ & $1.03[0.96,1.09]$ & & & & \\
\hline Mittal et al. 2017 & 25 & & 273 & 496 & $0.0 \%$ & $2.04[0.96,4.34]$ & & & & \\
\hline Nouh et al. 2017 & 67 & 134 & 719 & 1418 & $0.1 \%$ & $0.97[0.68,1.39]$ & & & + & \\
\hline Qiu et al. 2021 & 253 & 504 & 863 & 1691 & $0.4 \%$ & $0.97[0.79,1.18]$ & & & - & \\
\hline Suri et al. 2013 & 44 & 90 & 456 & 928 & $0.1 \%$ & $0.99[0.64,1.53]$ & & & & \\
\hline Vahidy et al. 2017 & 19699 & 38625 & 144837 & 280692 & $37.9 \%$ & $0.98[0.96,1.00]$ & & & & \\
\hline Wen et al. 2019 & 361 & 960 & 979 & 2513 & $0.7 \%$ & $0.94[0.81,1.10]$ & & & & \\
\hline Total $(95 \% \mathrm{Cl})$ & & 101054 & & 1189762 & $2 \quad 100.0 \%$ & $0.97[0.96,0.98]$ & & & & \\
\hline Total events & 50927 & & 603940 & & & & & & & \\
\hline Heterogeneity: $\mathrm{Chi}^{2}=18.4$ & 15, df $=13(P=$ & $0.14) ; 1^{2}=3$ & $30 \%$ & & & & 0.2 & 0.5 & & 5 \\
\hline Test for overall effect: $Z=$ & $4.84(P<0.00$ & & & & & & 0.2 & Favours No readmission & Favours Readmission & \\
\hline & Readmission & group $\mathrm{N}$ & No readmissio & on group & & Odds Ratio & & Odds $\mathrm{F}$ & Ratio & \\
\hline Study or Subgroup & Events & Total & Events & Total & Weight & M-H. Random, $95 \%$ Cl & & M-H. Rando & lom. $95 \% \mathrm{Cl}$ & \\
\hline Bhattacharya et al. 2011 & 8 & 22 & 79 & 170 & $0.1 \%$ & $0.66[0.26,1.65]$ & & & & \\
\hline Bjerkreim et al. 2018 & 95 & 200 & 743 & 1674 & $0.7 \%$ & $1.13[0.85,1.52]$ & & & & \\
\hline Boehme et al. 2018 & 24592 & 48125 & 168200 & 323462 & $31.9 \%$ & $0.96[0.95,0.98]$ & & 4 & & \\
\hline Crispo et al. 2018 & 3024 & 6205 & 222546 & 433477 & $14.9 \%$ & $0.90[0.86,0.95]$ & & -1 & & \\
\hline Han et al. 2015 & 806 & 1782 & 44072 & 97682 & $5.8 \%$ & $1.00[0.91,1.10]$ & & & - & \\
\hline Khanevski et al. 2018 & 16 & 33 & 822 & 1841 & $0.1 \%$ & $1.17[0.59,2.32]$ & & & & \\
\hline Kilkenny et al. 2013 & 101 & 215 & 1532 & 3113 & $0.8 \%$ & $0.91[0.69,1.21]$ & & & - & \\
\hline Lee et al. 2019 & 1836 & 4124 & 17819 & 40605 & $10.5 \%$ & $1.03[0.96,1.09]$ & & & $r$ & \\
\hline Mittal et al. 2017 & 25 & 35 & 273 & 496 & $0.1 \%$ & $2.04[0.96,4.34]$ & & & & \\
\hline Nouh et al. 2017 & 67 & 134 & 719 & 1418 & $0.5 \%$ & $0.97[0.68,1.39]$ & & & 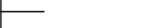 & \\
\hline Qiu et al. 2021 & 253 & 504 & 863 & 1691 & $1.5 \%$ & $0.97[0.79,1.18]$ & & - & - & \\
\hline Suri et al. 2013 & 44 & 90 & 456 & 928 & $0.3 \%$ & $0.99[0.64,1.53]$ & & & & \\
\hline Vahidy et al. 2017 & 19699 & 38625 & 144837 & 280692 & $30.5 \%$ & $0.98[0.96,1.00]$ & & & & \\
\hline Wen et al. 2019 & 361 & 960 & 979 & 2513 & $2.4 \%$ & $0.94[0.81,1.10]$ & & & & \\
\hline Total $(95 \% \mathrm{Cl})$ & & 101054 & & 1189762 & $100.0 \%$ & $0.97[0.94,0.99]$ & & () & & \\
\hline Total events & 50927 & & 603940 & & & & & & & \\
\hline $\begin{array}{l}\text { Heterogeneity: } \text { Tau }^{2}=0.00 ; \\
\text { Test for overall effect: } Z=2.6\end{array}$ & $\begin{array}{l}\mathrm{Ch}^{2}=18.45, \\
61(\mathrm{P}=0.009\end{array}$ & If $=13(P=$ & $0.14) ; I^{2}=30 \%$ & & & & 0.2 & 0.5 & 1 & \\
\hline
\end{tabular}

Figure 10 Influence of gender (female) on 30-day readmission in patients with ischemic stroke (Fixed effects model, Random effects model).

significant publication bias.

\section{Discussion}

To our knowledge, this is the first systematic review and meta-analysis of risk factors for 30-day readmission in patients with ischemic stroke. Although several systematic reviews and meta-analyses (8-10) have investigated the risk factors for readmission in stroke survivors, our current study possesses the following distinct strengths: (I) all the literature (including Chinese-language literature, Englishlanguage literature, gray literature, and references to relevant articles) as of April 30, 2021 was searched, and thus high-quality articles in the past 5 years were included in the analysis; (II) a total of 7 electronic databases (including Chinese-language databases) were searched, which were 


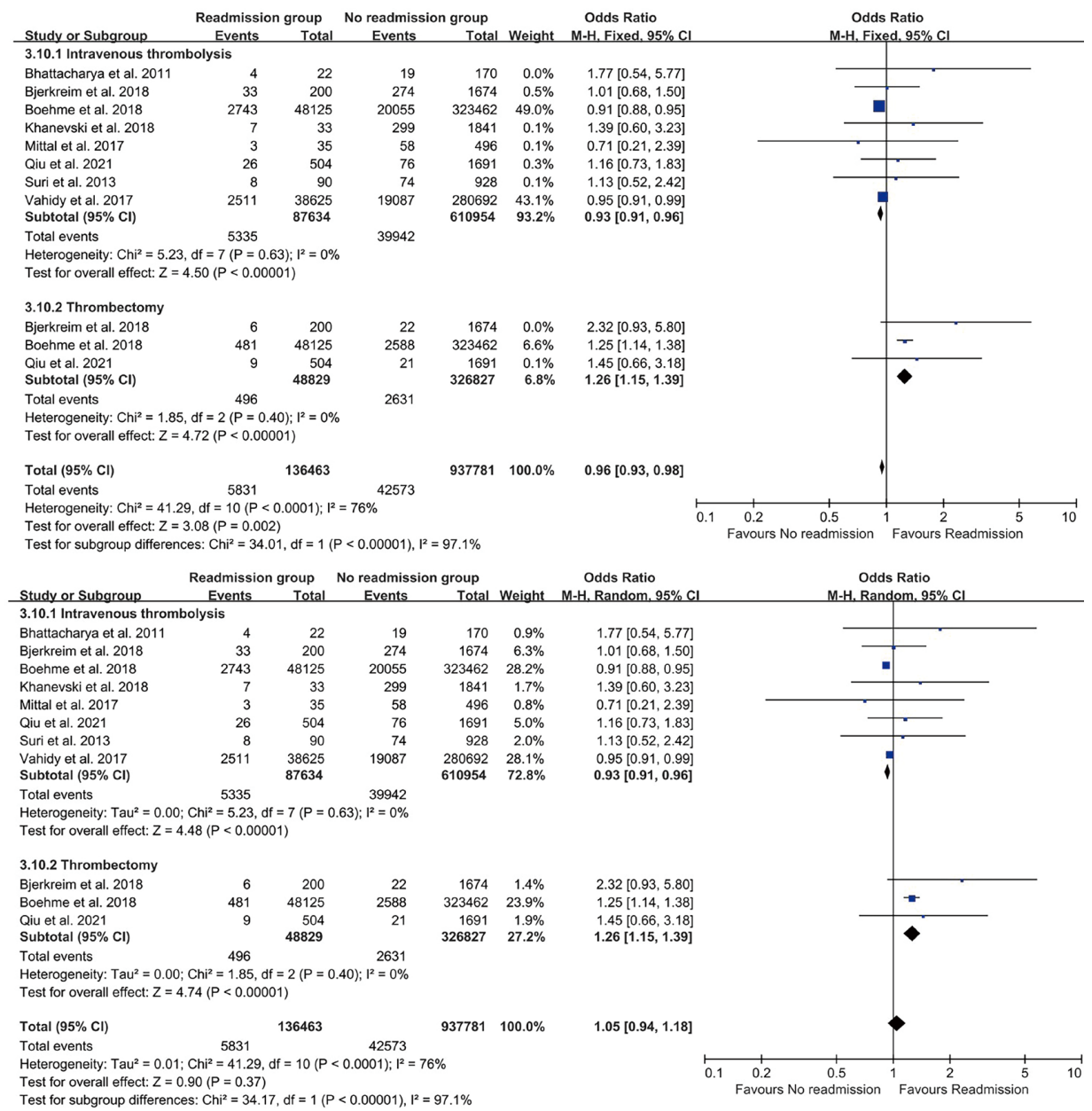

Figure 11 Influence of treatment modality on 30-day readmission in patients with ischemic stroke (Fixed effects model, Random effects model).

not examined in the previous meta-analyses; (III) only high-quality or moderate-quality studies were included for analysis, and some of these studies had large sample sizes, which increased the persuasiveness and scientific validity of our conclusions; and (IV) about 30 possible risk factors were considered, and detailed subgroup analyses were performed for some of the risk factors, with the results for some of these risk factors being reported for the first time.

Research on 30-day readmission in patients with ischemic stroke is a priority, and identifying the risk factors of readmissions is essential to implementing effective interventions and reducing the 30-day readmission rate. However, the exact risk factors remain controversial and have eluded exact study. The 30-day readmission rates of ischemic stroke survivors ranged from $1.41 \%$ to $27.64 \%$, with a mean value of $10.66 \%$ (SD $6.87 \%$ ). The results varied across countries and regions, with the highest reported 30-day readmission rate from China (11) and the lowest from the United States (12), which is consistent with the results of previous systematic reviews and meta-analyses (2,4,7,13-27). Many risk factors can contribute to 30-day readmission in patients with ischemic stroke. We screened about 30 of the most frequently mentioned risk factors in these 17 articles and finally identified 6 risk factors: history of stroke, diabetes mellitus, hypertension, atrial fibrillation, heart failure, and age. Among these, age was determined by descriptive analysis. Four risk factors were excluded, including hyperlipidemia, coronary heart disease, smoking, and gender, while 5 possible risk factors require further investigation, including duration of hospitalization, treatment modality, discharge disposition, health care payment model, and etiology. Intravenous thrombolysis and 


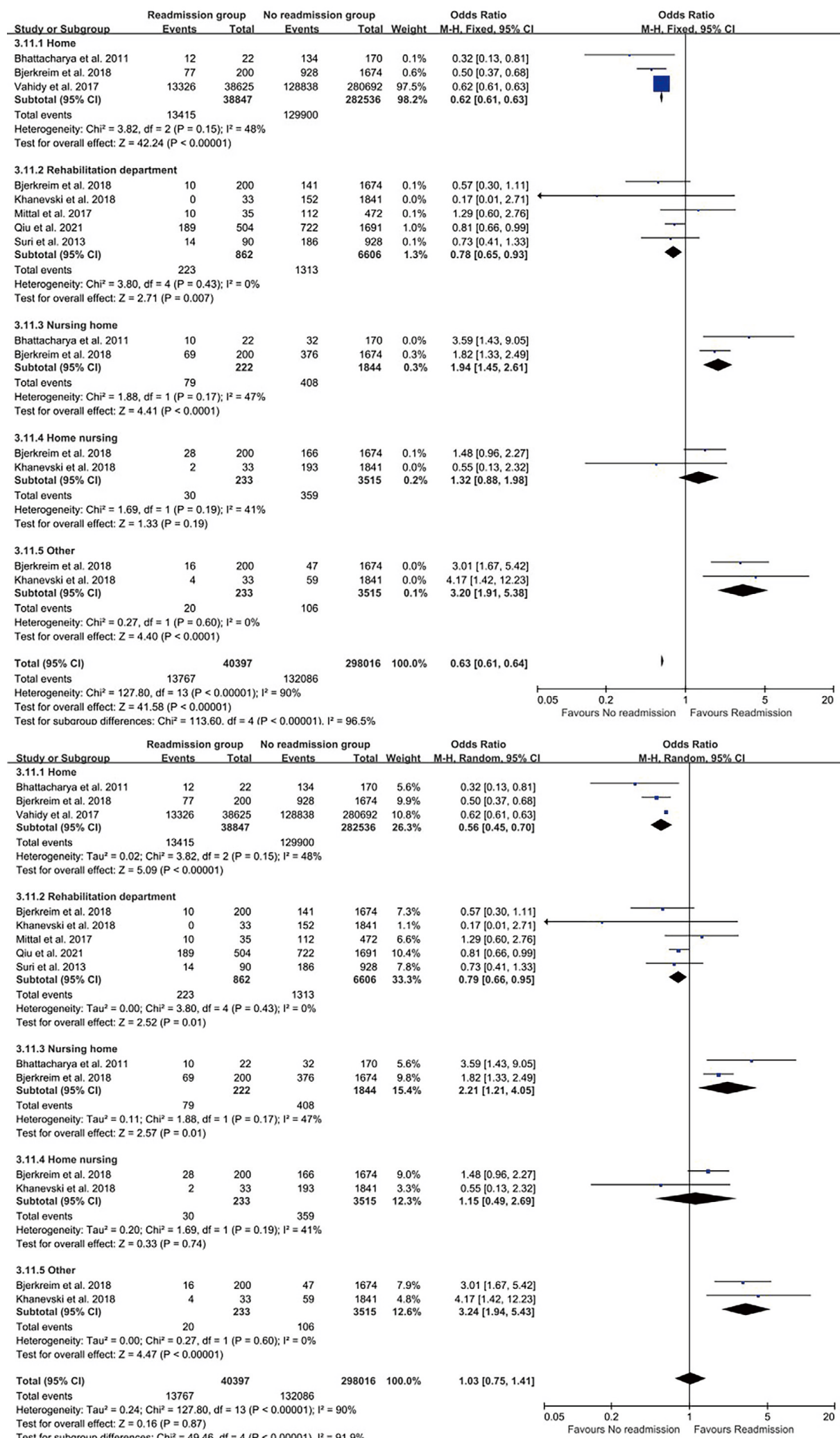

Figure 12 Influence of discharge destination on 30-day readmission in patients with ischemic stroke (Fixed effects model, Random effects model). 


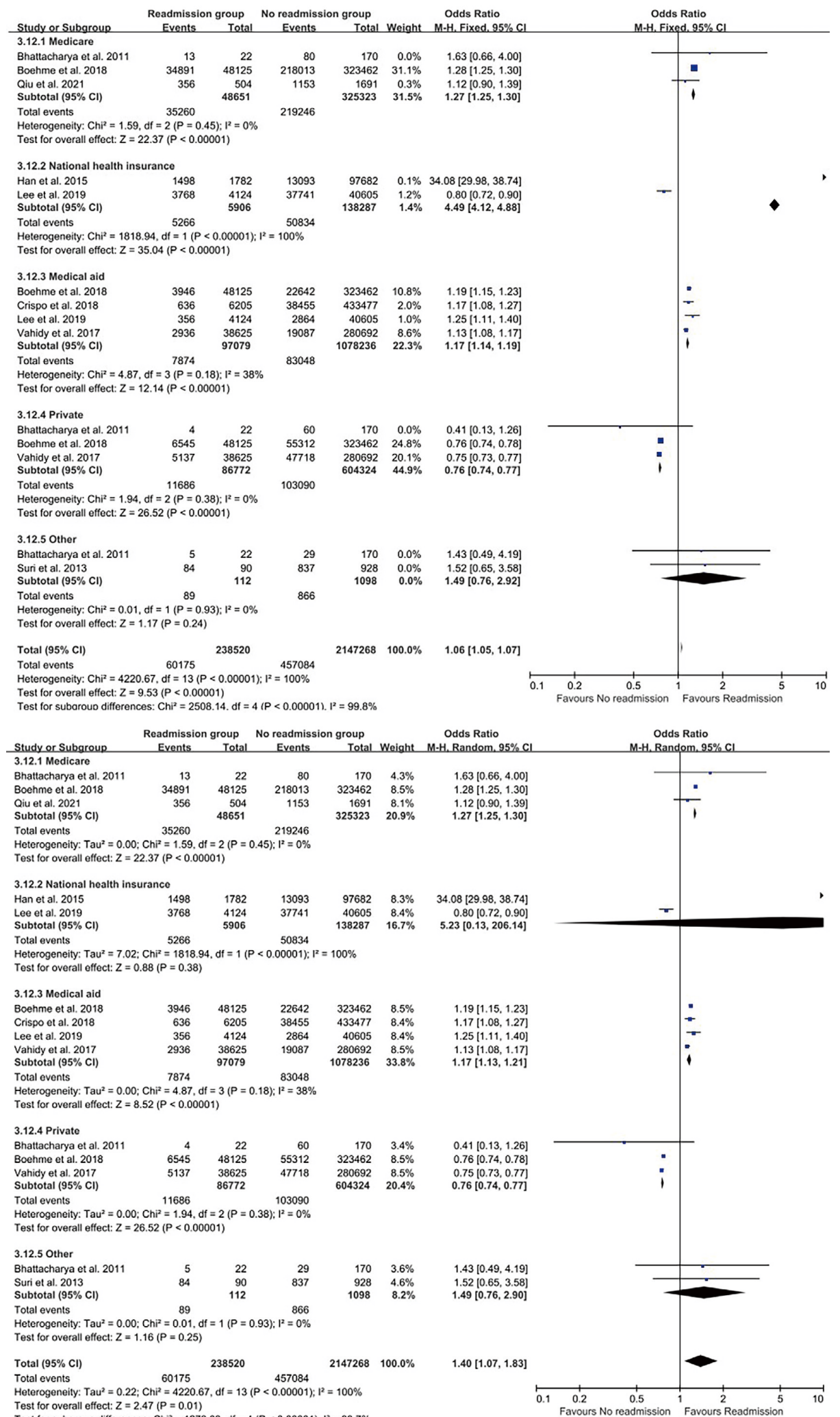

Figure 13 Influence of health care payment model on 30-day readmission in patients with ischemic stroke (Fixed effects model, Random effects model). 


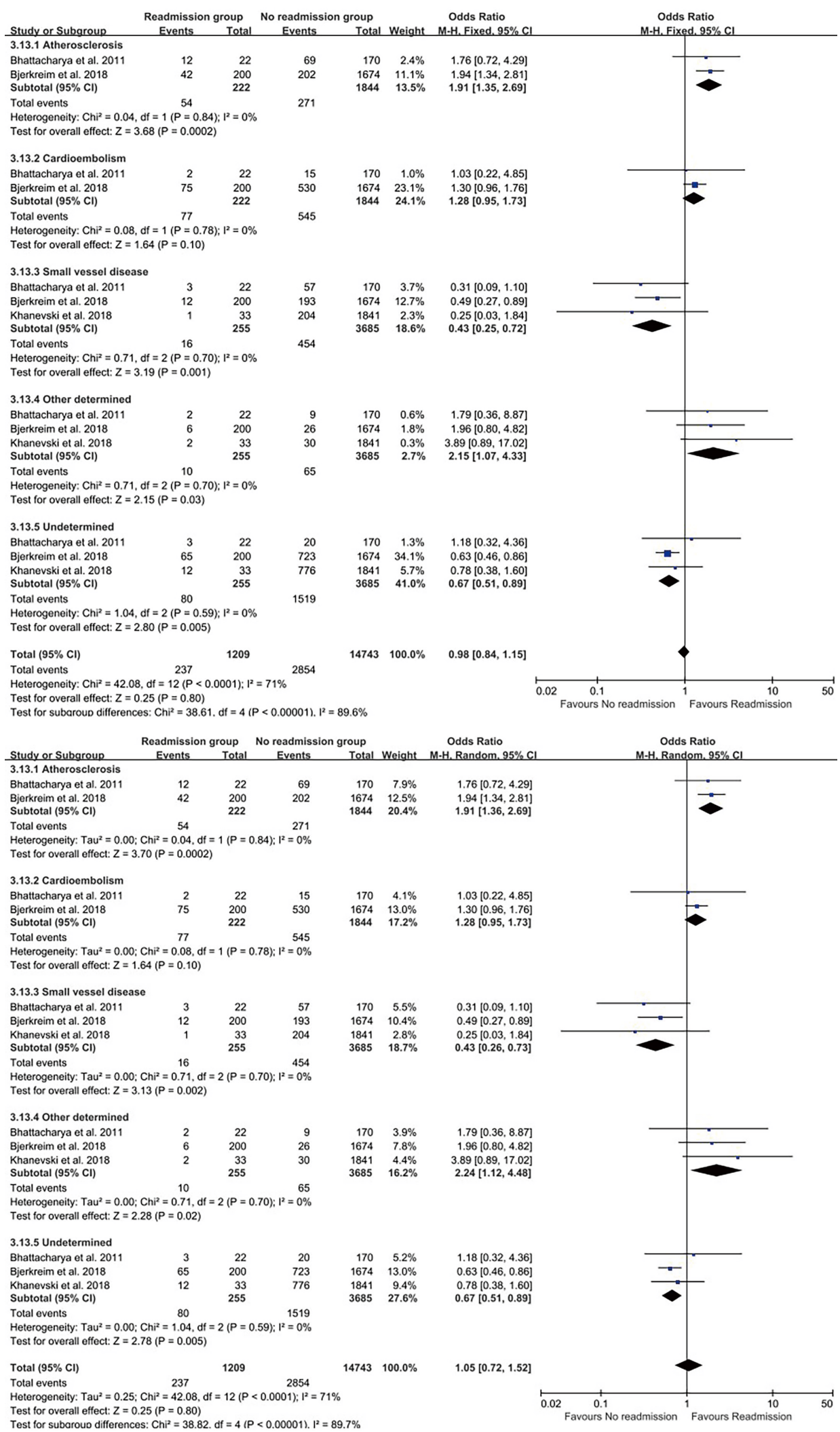

Figure 14 Influence of etiology on 30-day readmission in patients with ischemic stroke (Fixed effects model, Random effects model). 


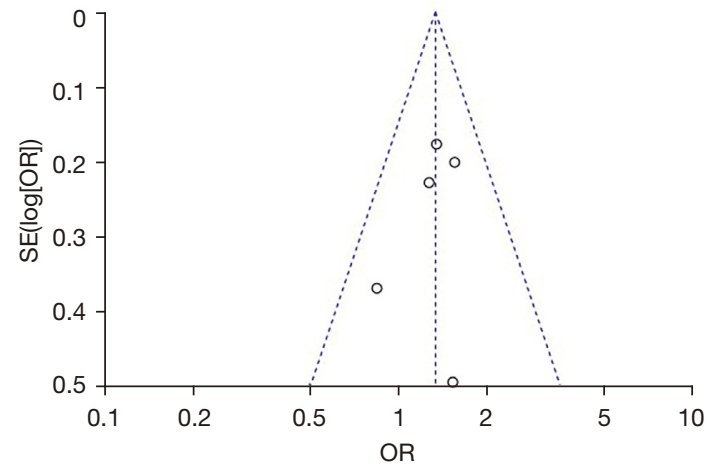

Figure 15 Publication bias of prior stroke.

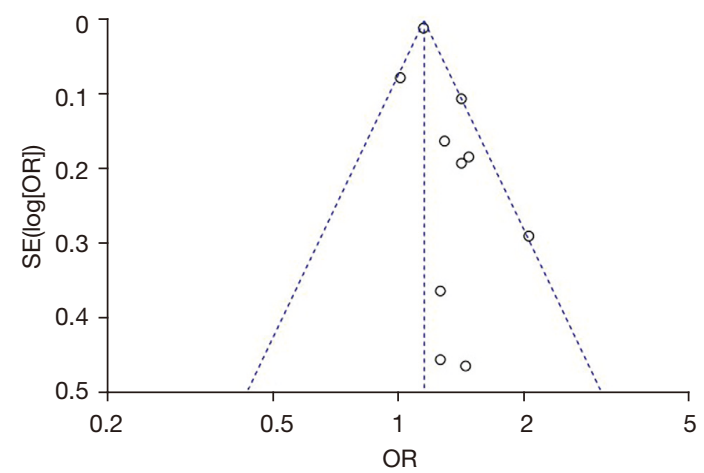

Figure 16 Publication bias of diabetes mellitus.

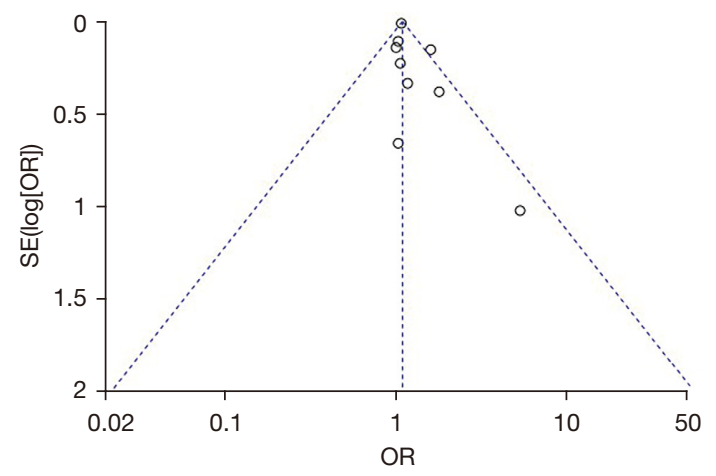

Figure 17 Publication bias of hypertension.

post-discharge rehabilitation showed protective effects. To our surprise, some of these risk factors have been shown to increase the risk of ischemic stroke, but our current study suggested that they did not increase the risk of 30-day readmission in patients with ischemic stroke.

We confirmed that a history of stroke, diabetes

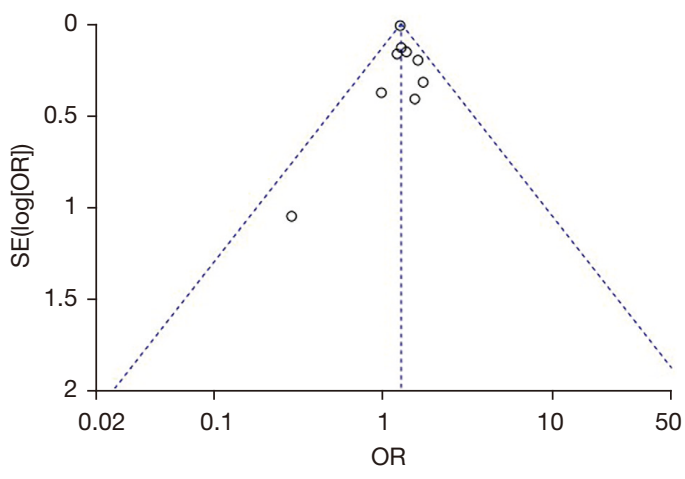

Figure 18 Publication bias of atrial fibrillation.

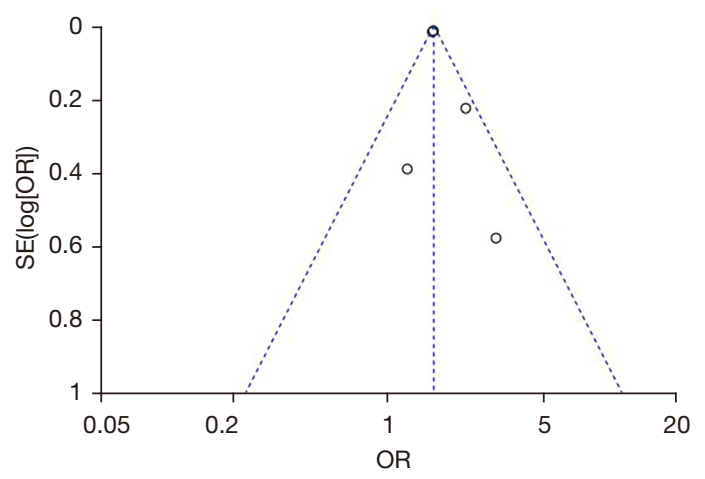

Figure 19 Publication bias of heart failure.

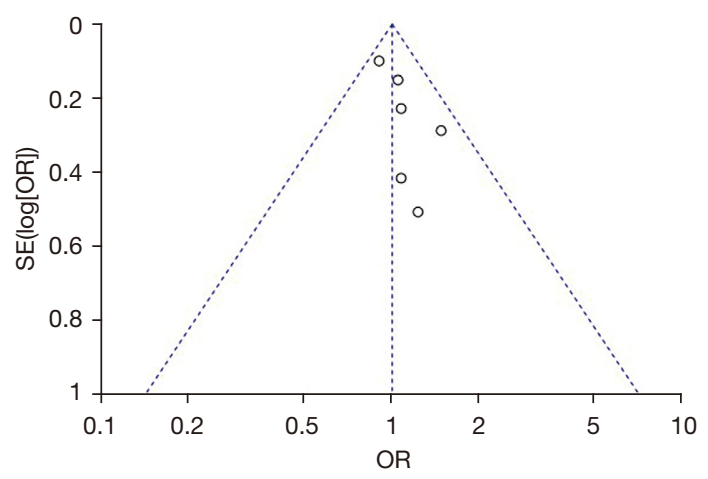

Figure 20 Publication bias of hyperlipidemia.

mellitus, hypertension, atrial fibrillation, or heart failure is a risk factor for 30-day readmission in patients with ischemic stroke. In our current analysis, "history of stroke" had different meanings across the included studies $(4,17,18,22,28-30)$ and was not explicitly defined in each study. Nevertheless, it could be definitively concluded 


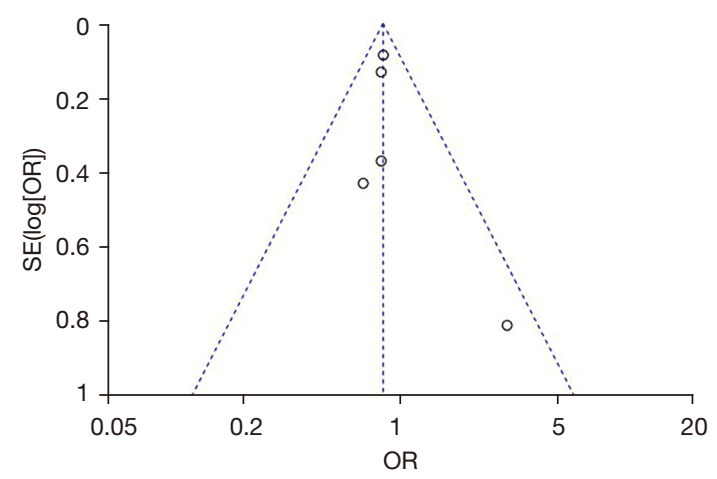

Figure 21 Publication bias of coronary heart disease.

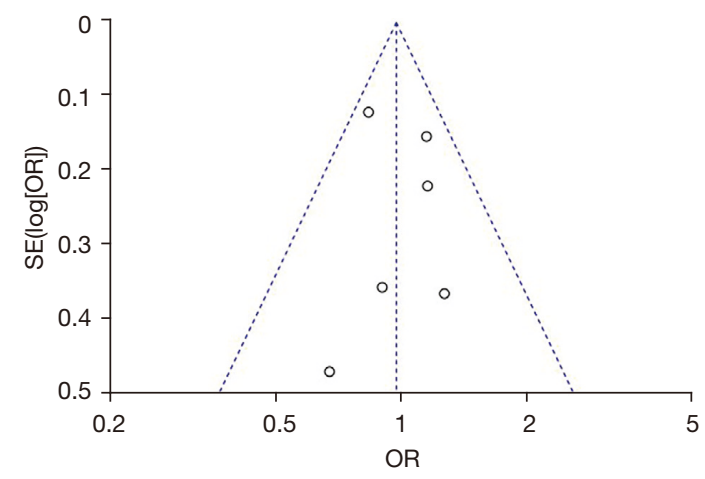

Figure 22 Publication bias of smoking.

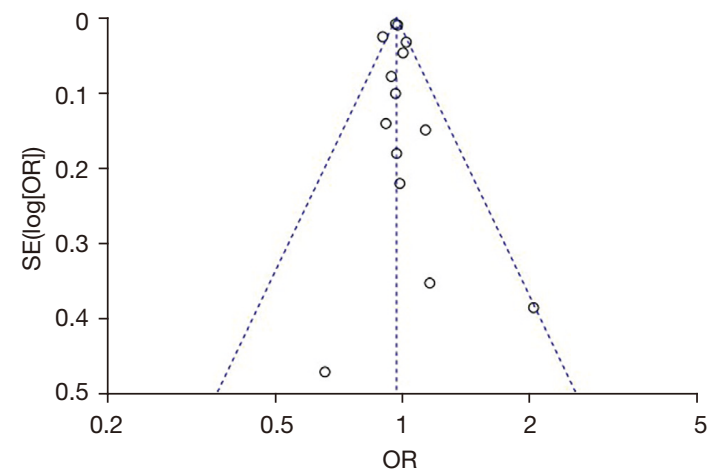

Figure 23 Publication bias of gender (female).

that patients with a history of stroke are more likely to be readmitted within 30 days after discharge than those who experienced stroke for the first time. The possible explanation for this is that patients with previous ischemic stroke episodes have more risk factors, such as worse vascular

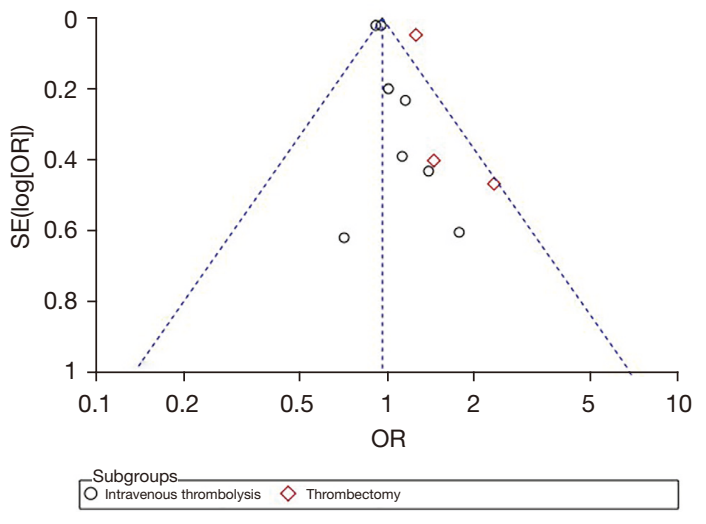

Figure 24 Publication bias of treatment modality.

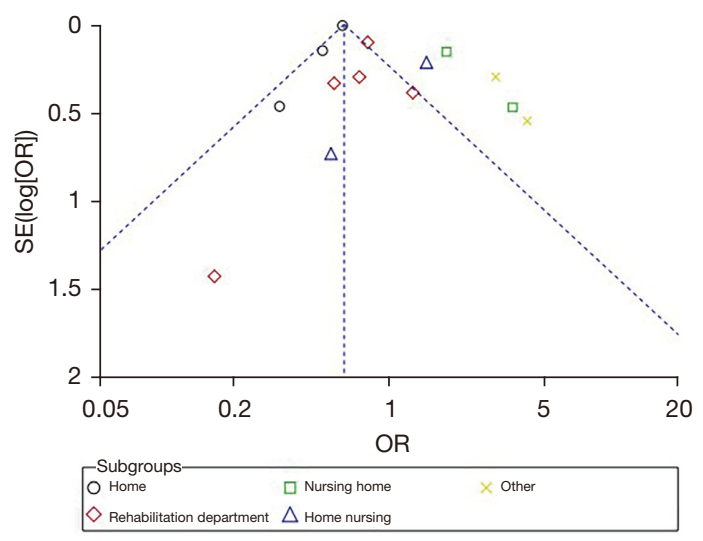

Figure 25 Publication bias of discharge destination.

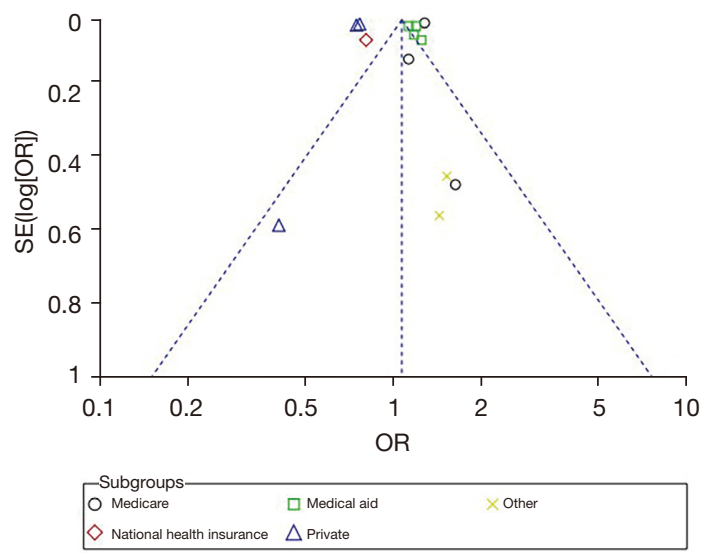

Figure 26 Publication bias of health care payment model. 


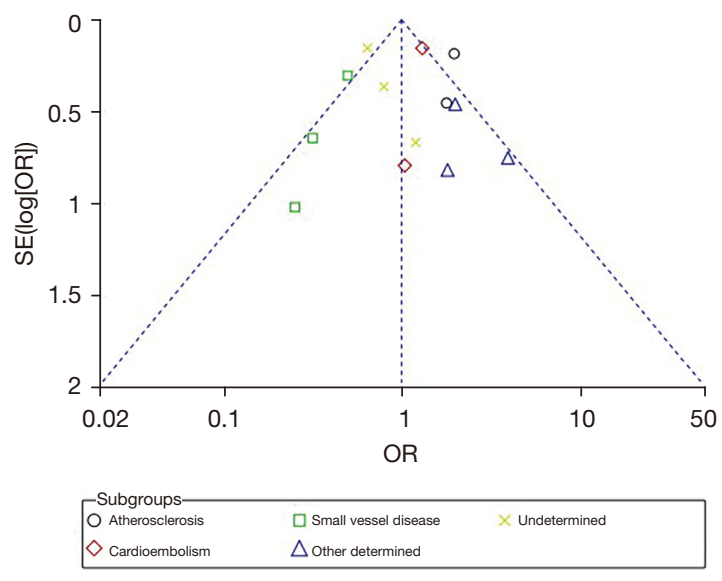

Figure 27 Publication bias of etiology.

condition, older age, or more comorbidities. However, it is unclear how a different, complex stroke history affects 30-day readmission. Whether diabetes mellitus, hypertension, atrial fibrillation, and heart failure are risk factors for 30-day readmission in patients with ischemic stroke remains controversial, even in some multicenter studies that had large sample sizes $(2,5,7,18,23,31,32)$. Our current analysis confirmed the associations of these 4 comorbidities with 30-day readmission. It is possible that when patients have these comorbidities, the inner walls of blood vessels are often damaged and blood is more likely to be in a hypercoagulable state, leading to thrombus formation or dislodgement of existing thrombus, which ultimately cause stroke recurrence. Stroke recurrence is the leading cause of 30-day readmission in patients with ischemic stroke $(2,4,7,17,24)$, and these comorbidities themselves may also directly lead to readmission $(15,16,24,31)$. In addition, the outcomes of recovery from these comorbidities were closely related to medications, outpatient follow-up, and patients' dietary habits and exercise. Furthermore, the patients' conditions and health care capacity varied from region to region, which may explain the inconsistent results across multiple studies. Therefore, for patients with ischemic stroke with a history of stroke, diabetes, hypertension, atrial fibrillation, and/or heart failure, tailored health care should be offered within 30 days after discharge, which may benefit more patients and lower the 30-day readmission rate.

Length of hospital stay and age have been explored in many studies, but the type of data reported varied widely across studies. Boehme et al., Vahidy et al., Lichtman et al., Qiu et al., and Han et al. $(5,7,18,28,33)$ presented the means and SDs of length of hospital stay. Bjerkreim et al.,
Wen et al., Mittal et al., Khanevski et al., and Kilkenny et al. $(4,11,30,34,35)$ reported the medians and quartiles, while Crispo et al. and Lee et al. $(12,36)$ performed subgroup comparisons. The same was true for age, with means and SD $(4,5,7,17,18,22,28,30,34)$, medians and quartiles $(11,35)$, and subgroup comparisons $(12,18,29,33,36)$ being reported in different articles, which proved problematic in allowing us to obtain accurate quantitative results via meta-analysis. We believe that advanced age is largely a risk factor for 30-day readmission, while the length of hospital stay is still a controversial factor. According to the studies performed by Bambhroliya et al. (3) $(\mathrm{n}=2,078,854)$ and by Hirayama et al. (37) ( $\mathrm{n}=620,788)$, advanced age is a risk factor for 30-day readmission in patients with ischemic stroke, which is consistent with the results of a study in China (2) that included 50,912 patients from 375 hospitals in 29 provinces. Hirayama et al. (37) noted that the 30-day readmission rate was significantly higher in patients older than 65 years compared with those younger than 65 years $(65-74$ years: OR $1.19,95 \%$ CI: $1.16-1.21 ; 75-84$ years: OR $1.29,95 \%$ CI: $1.27-1.31$; $>85$ years: OR $1.24,95 \%$ CI: $1.22-1.27$; all $\mathrm{P}<0.001)$. Nouh et al. (29) also showed that the 30-day readmission rate was higher in patients $>75$ years. Qureshi et al. (17) reported a $19 \%$ increase in the odds of readmission for each decade of older age. Compared to young and middle-aged patients, older adult patients had more comorbidities, as well as worse vascular condition, higher blood viscosity, and lower cardiac function, which could lead to hemodynamic deficiencies. As a result, patients with advanced age are more likely to be readmitted. A question then arises: what is the cutoff age for "advanced age"? There was no definite answer in our current analysis. In terms of length of hospital stay, some Korean and American studies $(7,12,33,36)$ suggested that the length of hospital stay affects the 30-day readmission in patients with ischemic stroke, which was corroborated by the results of a few prospective cohort studies $(32,38)$. Conversely, other studies from Norway (4), Australia (35), and China (28) reported opposing results. Unfortunately, some of the prospective studies with large sample sizes did not analyze the length of hospital stay $(4,37)$. A study by Wen et al. (2) showed that a length of hospital stay longer than 7 days was associated with a reduced risk of 30-day readmission. In another study by Wen et al. (11), however, the risk of 30-day readmission was lowest in patients hospitalized for about 10 days, and longer or shorter length of hospital stay increased the risk of readmission. The controversy over the length of hospital stay may be due to differences in region, 
sample size, study type, medical resources, and level of care, though one of the key considerations may be as follows: a longer length of hospital stay indicates that the disease is more severe and more difficult to treat; however, the patients receive more medical resources and high-quality care, which theoretically leads to better outcomes and thus lowers readmission rates. A shorter length of hospital stay may have the opposite effect. We hope that multicenter prospective studies with larger sample sizes can further clarify the role of the length of hospital stay.

In our current analysis, 4 risk factors were excluded, including hyperlipidemia, coronary heart disease, smoking, and gender. These 4 factors are known to be risk factors for the development of ischemic stroke. Interestingly, however, they were not associated with 30-day readmission in patients with ischemic stroke. In some previous studies $(4,11,32,39)$, abnormal lipid metabolism and heart-related diseases differed between two groups of patients, but the investigators did not analyze hyperlipidemia and coronary heart disease separately. In addition, although studies with large sample sizes performed by Crispo $e t a l$. and Lichtman et al. $(12,18)$ suggested that gender might increase the likelihood of 30-day readmission in patients with ischemic stroke, our analysis did find a basis for this association (1.3 million participants in 13 articles), which was supported by a prospective study performed by Terman et al. (32). This may be due to the fact that, within 30 days of discharge, patients have good medication compliance, their disease is effectively controlled, and acute conditions due to hyperlipidemia and coronary artery disease are relatively rare. For smoking and gender, a period of 30 days may be too short to reflect the impact these factors on readmission, and perhaps a longer period would have shown a difference. It is also possible that the included studies had some limitations in data analysis. For instance, gender was not analyzed in subgroups based on age or disease condition, and no accurate or specific data on smoking were collected.

Regretfully, we failed to analyze the impact of 5 possible risk factors on 30-day readmission in patients with ischemic stroke, including discharge disposition (home, rehabilitation facility, nursing home, home nursing, and others), health care payment model (Medicare, NHS, Medicaid, private insurance, and others), treatment modality (thrombolysis and thrombectomy), and etiology (atherosclerosis, cardiogenic cerebral embolism, small vessel disease, other definite causes, and other unknown causes). Nevertheless, we were the first to perform a detailed and systematic subgroup analysis of these factors. In fact, we performed subgroup analyses for each of these factors, but the literature was insufficient due to heterogeneity. Only intravenous thrombolysis and post-discharge rehabilitation had valuable outcomes in that they were protective factors for 30-day readmission in patients with ischemic stroke. This may be because patients who complete intravenous thrombolysis are those who are sent to the hospital promptly after disease onset for treatment, where they receive excellent medical care and have good outcomes and significant recovery immediately after completion of thrombolysis, which may eliminate the impact of inadequate blood supply to the brain. Therefore, we suggest that active intravenous thrombolysis should be carried out for patients with ischemic stroke who arrive in hospital within the treatment time window according to the standard operating requirements to reduce the risk of 30-day readmission. Postdischarge rehabilitation can help patients effectively control various risk factors, receive more health care, and reduce the risk of 30-day readmission. According to Andrews et al. (40), compared with low- and medium-intensity therapy, highintensity therapy (physical therapy, occupational therapy, and speech therapy) can lower the 30-day readmission rate. However, patients who receive higher-intensity therapy may have more comorbidities and greater illness severity relative to those who receive lower-intensity therapy. We suggest that medical staff should strengthen health education, emphasize the importance of active rehabilitation when patients are discharged from hospital and in outpatient treatment, and provide personalized rehabilitation guidance according to each patient's condition.

Our research also has some limitations. First, some of the factors [e.g., alcohol consumption, marriage, infection, National Institutes of Health Stroke Scale (NIHSS) score, nasogastric tube feeding, and indwelling catheter] were not subjected to meta-analysis because of the limited amount of included literature (41), but they were still very common. Although they have been investigated in a few multicenter studies with large sample sizes, more studies are still needed. Second, some potential risk factors were not included because the number of prospective observational or interventional studies focusing on the same factor was too small for a meta-analysis or a descriptive analysis. Nevertheless, we took these studies into account as much as possible in the analysis. All the studies included in this analysis were case-control studies, so causality cannot be inferred. In addition, recall bias may exist. Third, most of the included articles were from the United States, which 
might have had an impact on the study results due to the differences in cultural background, health care services, and research capacity. Some of the included articles did not strictly meet the inclusion or exclusion criteria, which might have also affected our conclusions. Fourth, some of the databases were not searched due to the limited resources of the research institutions. However, the databases used in our current analysis are large international databases that are commonly used in academic research. In particular, we searched Chinese databases, which ensures that the vast majority of the relevant literature was retrieved.

\section{Conclusions}

The 30-day readmission rate remains high in ischemic stroke survivors, ranging from $1.41 \%$ to $27.64 \%$. The results varied across countries and regions, with the highest reported 30-day readmission rate from China and the lowest from the United States. Special attention should be paid to patients with a history of stroke, diabetes, hypertension, atrial fibrillation, heart failure, and/or advanced age. Timely intravenous thrombolysis can alleviate the disease, and post-discharge rehabilitation should be encouraged. These interventions help to reduce 30-day readmissions and benefit more patients. In contrast, interventions based on hyperlipidemia, coronary heart disease, smoking status, or gender may not improve the current situation. Additional research is needed on the length of hospital stay, treatment modality, discharge disposition, health care payment model, and etiology to explore their impact on 30-day readmission in patients with ischemic stroke.

\section{Acknowledgments}

Funding: This work was supported by West China Nursing Discipline Development Special Fund Project, Sichuan University (grant No. HXHL19004). The funding bodies were not involved in any aspects of the study.

\section{Footnote}

Reporting Checklist: The authors have completed the PRISMA reporting checklist. Available at https://dx.doi. org/10.21037/apm-21-2884

Conflicts of Interest: All authors have completed the ICMJE uniform disclosure form (available at https://dx.doi. org/10.21037/apm-21-2884). The authors have no conflicts of interest to declare.

Ethical Statement: The authors are accountable for all aspects of the work in ensuring that questions related to the accuracy or integrity of any part of the work are appropriately investigated and resolved.

Open Access Statement: This is an Open Access article distributed in accordance with the Creative Commons Attribution-NonCommercial-NoDerivs 4.0 International License (CC BY-NC-ND 4.0), which permits the noncommercial replication and distribution of the article with the strict proviso that no changes or edits are made and the original work is properly cited (including links to both the formal publication through the relevant DOI and the license). See: https://creativecommons.org/licenses/by-nc-nd/4.0/.

\section{References}

1. GBD 2016 Stroke Collaborators. Global, regional, and national burden of stroke, 1990-2016: a systematic analysis for the Global Burden of Disease Study 2016. Lancet Neurol 2019;18:439-58.

2. Wen T, Liu B, Wan X, et al. Risk factors associated with 31-day unplanned readmission in 50,912 discharged patients after stroke in China. BMC Neurol 2018;18:218.

3. Bambhroliya AB, Donnelly JP, Thomas EJ, et al. Estimates and Temporal Trend for US Nationwide 30-Day Hospital Readmission Among Patients With Ischemic and Hemorrhagic Stroke. JAMA Netw Open 2018;1:e181190.

4. Bjerkreim AT, Khanevski AN, Selvik HA, et al. The Impact of Ischaemic Stroke Subtype on 30-day Hospital Readmissions. Stroke Res Treat 2018;2018:7195369.

5. Boehme AK, Kulick ER, Canning M, et al. Infections Increase the Risk of 30-Day Readmissions Among Stroke Survivors. Stroke 2018;49:2999-3005.

6. Zuckerman RB, Sheingold SH, Epstein AM. The Hospital Readmissions Reduction Program. N Engl J Med 2016;375:494.

7. Vahidy FS, Donnelly JP, McCullough LD, et al. Nationwide Estimates of 30-Day Readmission in Patients With Ischemic Stroke. Stroke 2017;48:1386-8.

8. Rao A, Barrow E, Vuik S, et al. Systematic Review of Hospital Readmissions in Stroke Patients. Stroke Res Treat 2016;2016:9325368.

9. Zhong W, Geng N, Wang P, et al. Prevalence, causes and 
risk factors of hospital readmissions after acute stroke and transient ischemic attack: a systematic review and metaanalysis. Neurol Sci 2016;37:1195-202.

10. Lichtman JH, Leifheit-Limson EC, Jones SB, et al. Predictors of hospital readmission after stroke: a systematic review. Stroke 2010;41:2525-33.

11. Wen TC, Liu BY, Zhang YN. Risk factors for unplanned readmission in ischemic stroke survivors within 31 days: a random forest algorithm research. Chinese Journal of Evidence-Based Medicine 2019;19:532-8.

12. Crispo JAG, Thibault DP, Fortin Y, et al. Association between medication-related adverse events and nonelective readmission in acute ischemic stroke. BMC Neurol 2018;18:192.

13. Fehnel CR, Lee Y, Wendell LC, et al. Post-Acute Care Data for Predicting Readmission After Ischemic Stroke: A Nationwide Cohort Analysis Using the Minimum Data Set. J Am Heart Assoc 2015;4:e002145.

14. Hung LC, Sung SF, Hu YH. A Machine Learning Approach to Predicting Readmission or Mortality in Patients Hospitalized for Stroke or Transient Ischemic Attack. Applied Sciences-Basel 2020;10:6337.

15. Yousufuddin M, Bartley AC, Alsawas M, et al. Impact of Multiple Chronic Conditions in Patients Hospitalized with Stroke and Transient Ischemic Attack. J Stroke Cerebrovasc Dis 2017;26:1239-48.

16. Zhong WB. The risk evaluation of readmission of acute ischemic stroke and transient ischemic attack. Shangdong University, 2017.

17. Qureshi AI, Baskett WI, Huang W, et al. Acute Ischemic Stroke and COVID-19: An Analysis of 27676 Patients. Stroke 2021;52:905-12.

18. Lichtman JH, Leifheit-Limson EC, Jones SB, et al. Preventable readmissions within 30 days of ischemic stroke among Medicare beneficiaries. Stroke 2013;44:3429-35.

19. Nahab F, Takesaka J, Mailyan E, et al. Avoidable 30day readmissions among patients with stroke and other cerebrovascular disease. Neurohospitalist 2012;2:7-11.

20. Lin HJ, Chang WL, Tseng MC. Readmission after stroke in a hospital-based registry: risk, etiologies, and risk factors. Neurology 2011;76:438-43.

21. Li HW, Yang MC, Chung KP. Predictors for readmission of acute ischemic stroke in Taiwan. J Formos Med Assoc 2011;110:627-33.

22. Bhattacharya P, Khanal D, Madhavan R, et al. Why do ischemic stroke and transient ischemic attack patients get readmitted? J Neurol Sci 2011;307:50-4.

23. Burke JF, Skolarus LE, Adelman EE, et al. Influence of hospital-level practices on readmission after ischemic stroke. Neurology 2014;82:2196-204.

24. Lakshminarayan K, Schissel C, Anderson DC, et al. Fiveyear rehospitalization outcomes in a cohort of patients with acute ischemic stroke: Medicare linkage study. Stroke 2011;42:1556-62.

25. Thompson MP, Zhao X, Bekelis K, et al. Regional Variation in 30-Day Ischemic Stroke Outcomes for Medicare Beneficiaries Treated in Get With The Guidelines-Stroke Hospitals. Circ Cardiovasc Qual Outcomes 2017;10:e003604.

26. Allen A, Barron T, Mo A, et al. Impact of Neurological Follow-Up on Early Hospital Readmission Rates for Acute Ischemic Stroke. Neurohospitalist 2017;7:127-31.

27. Suri MF, Qureshi AI. Readmission within 1 month of discharge among patients with acute ischemic stroke: results of the University HealthSystem Consortium Stroke Benchmarking study. J Vasc Interv Neurol 2013;6:47-51.

28. Qiu X, Xue X, Xu R, et al. Predictors, causes and outcome of 30-day readmission among acute ischemic stroke. Neurol Res 2021;43:9-14.

29. Nouh AM, McCormick L, Modak J, et al. High Mortality among 30-Day Readmission after Stroke: Predictors and Etiologies of Readmission. Front Neurol 2017;8:632.

30. Mittal MK, Rabinstein AA, Mandrekar J, et al. A population-based study for 30-d hospital readmissions after acute ischemic stroke. Int J Neurosci 2017;127:305-13.

31. Elgendy IY, Omer MA, Kennedy KF, et al. 30-Day Readmissions After Endovascular Thrombectomy for Acute Ischemic Stroke. JACC Cardiovasc Interv 2018;11:2414-24.

32. Terman SW, Reeves MJ, Skolarus LE, et al. Association Between Early Outpatient Visits and Readmissions After Ischemic Stroke. Circ Cardiovasc Qual Outcomes 2018;11:e004024.

33. Han KT, Kim SJ, Jang SI, et al. Positive correlation between care given by specialists and registered nurses and improved outcomes for stroke patients. J Neurol Sci 2015;353:137-42.

34. Khanevski AN, Bjerkreim AT, Novotny V, et al. Thirtyday recurrence after ischemic stroke or TIA. Brain Behav 2018;8:e01108.

35. Kilkenny MF, Longworth M, Pollack M, et al. Factors associated with 28-day hospital readmission after stroke in Australia. Stroke 2013;44:2260-8.

36. Lee SA, Park EC, Shin J, et al. Patient and hospital factors associated with 30-day unplanned readmission in patients 
with stroke. J Investig Med 2019;67:52-8.

37. Hirayama A, Goto T, Faridi MK, et al. Age-related differences in the rate and diagnosis of 30-day readmission after hospitalization for acute ischemic stroke. Int J Stroke 2018;13:717-24.

38. Shah SV, Corado C, Bergman D, et al. Impact of Poststroke Medical Complications on 30-Day Readmission Rate. J Stroke Cerebrovasc Dis 2015;24:1969-77.

39. Jin P, Matos Diaz I, Stein L, et al. Intermediate risk of cardiac events and recurrent stroke after stroke admission in young adults. Int J Stroke 2018;13:576-84.

40. Andrews AW, Li D, Freburger JK. Association of Rehabilitation Intensity for Stroke and Risk of Hospital Readmission. Phys Ther 2015;95:1660-7.

41. Lekoubou A, Bishu KG, Ovbiagele B. Influence of a Comorbid Diagnosis of Seizure on 30-Day Readmission Rates Following Hospitalization for an Index Stroke. J Stroke Cerebrovasc Dis 2020;29:104479.
Cite this article as: Deng Z, Wu X, Hu L, Li M, Zhou M, Zhao L, Yang R. Risk factors for 30-day readmission in patients with ischemic stroke: a systematic review and meta-analysis. Ann Palliat Med 2021;10(10):11083-11105. doi: 10.21037/apm-212884 